\title{
Geometrical Classification of Self-Similar Motion of Two- Dimensional Three Point Vortex System by Deviation Curvature on Jacobi Field
}

\author{
Yuma Hirakui $\mathbb{D D}^{1}$ and Takahiro Yajima ${ }^{2}$ \\ ${ }^{1}$ Division of Engineering and Agriculture, Graduate School of Regional Development and Creativity, Utsunomiya University, \\ Utsunomiya 321-8585, Japan \\ ${ }^{2}$ Course of Mechanical Systems Engineering, School of Engineering, Utsunomiya University, Utsunomiya 321-8585, Japan \\ Correspondence should be addressed to Yuma Hirakui; mc206750@cc.utsunomiya-u.ac.jp
}

Received 20 March 2021; Accepted 13 May 2021; Published 28 October 2021

Academic Editor: Manuel De Le n

Copyright (c) 2021 Yuma Hirakui and Takahiro Yajima. This is an open access article distributed under the Creative Commons Attribution License, which permits unrestricted use, distribution, and reproduction in any medium, provided the original work is properly cited.

In this study, we geometrically analyze the relation between a point vortex system and deviation curvatures on the Jacobi field. First, eigenvalues of deviation curvatures are calculated from relative distances of point vortices in a three point vortex system. Afterward, based on the assumption of self-similarity, time evolutions of eigenvalues of deviation curvatures are shown. The self-similar motions of three point vortices are classified into two types, expansion and collapse, when the relative distances vary monotonously. Then, we find that the eigenvalues of self-similarity are proportional to the inverse fourth power of relative distances. The eigenvalues of the deviation curvatures monotonically convergent to zero for expansion, whereas they monotonically diverge for collapse, which indicates that the strengths of interactions between point vortices related to the time evolution of spatial geometric structure in terms of the deviation curvatures. In particular, for collapse, the collision point becomes a geometric singularity because the eigenvalues of the deviation curvature diverge. These results show that the selfsimilar motions of point vortices are classified by eigenvalues of the deviation curvature. Further, nonself-similar expansion is numerically analyzed. In this case, the eigenvalues of the deviation curvature are nonmonotonous but converge to zero, suggesting that the motion of the nonself-similar three point vortex system is also classified by eigenvalues of the deviation curvature.

\section{Introduction}

Geometric theories are often applied to analyze dynamical system behavior. The relationship between a dynamical system and geometry is such that the Euler-Lagrange equation corresponds to the geodesic equation as a second-order differential equation. An invariant theory of a system of second-order differential equations has been established by Kosambi [1], Cartan and Kosambi [2], and Chern [3] (KCC theory). From the KCC theory, there are five geometric invariants. The first invariant is related to the existence of external force. The second invariant is a deviation curvature; eigenvalues of a deviation curvature define the Jacobi stability of whole trajectories. The third invariant is obtained from the differential of a deviation curvature. The fourth invariant is called the Riemann curvature and is associated with deviation curvature. The fifth invariant is called the Douglas tensor.

In previous studies, the theory of the Jacobi field has been applied to various dynamical systems, and concrete phenomena in biology, oscillating system, geophysics, astrophysics, and chaos models have been analyzed using the KCC invariants [4-28]. In biology, symbiogenesis modeled by the Volterra-Hamilton system has been studied in the Jacobi field [6]. From this analysis, a characteristic of the model has been clarified by the invariants. For the oscillating system, the study of van der Pol has compared the Jacobi and linear stabilities $[12,14]$, and the study of overhead cranes 
has investigated the relation between the Jacobi stability and Hopf bifurcation [15]. In geophysics, the Lorenz and Rikitake systems have been studied. The Jacobi stability of the Lorenz system has been analyzed around equilibrium points [17]. Moreover, the nonperiodic behavior of the Lorenz and Rikitake systems has been discussed on the basis of the third invatiant $[21,22]$. In astrophysics, the KCC theory has been applied to braneworld models [23]. For chaos models, the Rabinovich-Fabrikant model has been examined from the viewpoint of the KCC theory [27].

In the theory of dynamical systems, it is known that the Hamilton formalism was used to model a behavior of system. From the viewpoint of the Jacobi field theory, geometric structures of the Hamilton system have only been studied in terms of a general form [29]. The relationship between geometric objects and the behavior of specific phenomena remains unclear. As a concrete Hamilton system, the motion of point vortices in fluid dynamics has been studied widely [30-36]. Particularly, a two point vortex system can be classified into two types, translation and rigid rotation. In a three point vortex system, chaotic behavior does not develop because the system is integrable [32]. Moreover, four kinds of motion, translation, rigid rotation, expansion, and collapse, are found in three point vortices that satisfy a condition of self-similarity. Here, the self-similarity means that the similarity of initial positions of point vortices is retained whenever. Translation and rigid rotation are classified by circulation conditions, whereas expansion and collapse are determined by circulation conditions and initial positions $[30,33,35]$. In such a point vortex system, equations of motion are expressed by a system of first-order differential equations, which means that the theory of the Jacobi field can be applied to the point vortex system by differentiating the equations of motion. Therefore, geometric objects, such as deviation curvatures can be obtained from the secondorder differential equations of the point vortex system. Then, we consider the Jacobi field of the three point vortex system and discuss a classification of motion based on the deviation curvature.

The remainder of this article is structured as follows. In Section 2, we review the KCC theory. In Section 3, we explain the motions of point vortices and their conditions of self-similarity. In Section 4, we calculate eigenvalues of deviation curvatures on self-similarity and discuss a classification of the motions of point vortices by the eigenvalues of the deviation curvature. Finally, Section 5 concludes this study.

\section{KCC Theory and Jacobi Stability}

In this section, we review the KCC theory and Jacobi stability based on $[4,12,14,37,38]$. Notably, the Einstein convention is used throughout this section. Let $M$ be a smooth $n$ -dimensional manifold, and $(T M, \pi, M)$ be a tangent bundle. A projection $\pi: T M \longrightarrow M$ is a mapping from $T M$ to $M$. Local coordinates on $M$ are denoted by $x^{i}$, where $i=1, \cdots, n$ . Local coordinates on TM are defined by $\left(x^{i}, \dot{x}^{i}\right)$, where $t$ is the time parameter, and $\dot{x}^{i}=d x^{i} / d t$. We consider the follow- ing system of second-order differential equations:

$$
\ddot{x}^{i}+2 G^{i}(x, \dot{x})=0,
$$

where $G^{i}(x, \dot{x})$ is a smooth function. The first invariant $E^{i}$ is given by

$$
E^{i}=2 G^{i}(x, \dot{x})-N_{j}^{i} \dot{x}^{j}
$$

where $N_{j}^{i}$ is the coefficient of nonlinear connection:

$$
N_{j}^{i}=\frac{\partial G^{i}}{\partial \dot{x}^{j}}
$$

A trajectory $x^{i}(t)$ of Equation (1) is perturbed to a trajectory $\bar{x}^{i}=x^{i}+u^{i} \delta \tau$, where $u^{i}$ is a vector field, and $\delta \tau$ is an infinitesimal parameter. From Equation (1), a variation equation is obtained as follows $\delta \tau \longrightarrow 0$ :

$$
\ddot{u}^{i}+2 \frac{\partial G^{i}}{\partial \dot{x}^{j}} \dot{u}^{j}+2 \frac{\partial G^{i}}{\partial x^{j}} u^{j}=0
$$

Then, the KCC-covariant derivative is defined by

$$
\frac{D u^{i}}{d t}=\dot{u}^{i}+N_{j}^{i} u^{j}
$$

Using (5), Equation (4) is rewritten as follows:

$$
\frac{D^{2} u^{i}}{d t^{2}}=P_{j}^{i} u^{j}
$$

where $P_{j}^{i}$ is the second invariant and is called a deviation curvature tensor

$$
P_{j}^{i}=-2 \frac{\partial G^{i}}{\partial x^{j}}+\frac{\partial N_{j}^{i}}{\partial x^{k}} \dot{x}^{k}-2 G_{j k}^{i} G^{k}+N_{k}^{i} N_{j}^{k} .
$$
by

Here, $G_{j k}^{i}$ is a coefficient of the Berwald connection given

$$
G_{j k}^{i}=\frac{\partial N_{j}^{i}}{\partial \dot{x}^{k}}
$$

The third, fourth, and fifth invariants are defined, respectively, as follows:

$$
\begin{gathered}
B_{j k}^{i}=\frac{1}{3}\left(\frac{\partial P_{j}^{i}}{\partial \dot{x}^{k}}-\frac{\partial P_{k}^{i}}{\partial \dot{x}^{j}}\right), \\
B_{l j k}^{i}=\frac{\partial B_{j k}^{i}}{\partial \dot{x}^{l}}, \\
D_{j k l}^{i}=\frac{\partial^{3} G^{i}}{\partial \dot{x}^{j} \partial \dot{x}^{k} \partial \dot{x}^{l}}
\end{gathered}
$$

The deviation curvature tensor $P_{j}^{i}$ gives the stability of 
whole trajectories via the following definition $[12,14]$ : The trajectories of the system (1) are Jacobi stable if and only if the real parts of the eigenvalues of $P_{j}^{i}$ are strictly negative everywhere, and Jacobi unstable otherwise.

\section{Review of Point Vortex and Its Self-Similarity}

In this section, we review a motion of point vortices that satisfies a self-similarity condition based on previous studies [30-34]. The self-similar motion means that the similarity of initial positions of point vortices is retained whenever, namely, the proportion of relative distances are constant. Moreover, we mention the linear stability of a motion of point vortices using relative distances at the end of this section. First, coordinates of three point vortices are denoted by $\left(x^{1}, y^{1}\right),\left(x^{2}, y^{2}\right)$, and $\left(x^{3}, y^{3}\right)$. Their respective relative distances are defined as follows:

$$
\begin{aligned}
& s^{1}=\sqrt{\left(x^{2}-x^{3}\right)^{2}+\left(y^{2}-y^{3}\right)^{2}}, \\
& s^{2}=\sqrt{\left(x^{3}-x^{1}\right)^{2}+\left(y^{3}-y^{1}\right)^{2}}, \\
& s^{3}=\sqrt{\left(x^{1}-x^{2}\right)^{2}+\left(y^{1}-y^{2}\right)^{2}} .
\end{aligned}
$$

Generally, equations of motions of point vortices are obtained from complex velocity potential. From [32], the equations of motion of three point vortices are given by

$$
\begin{aligned}
& \frac{d x^{1}}{d t}=-\frac{1}{2 \pi}\left(\frac{\Gamma_{2}\left(y^{1}-y^{2}\right)}{\left(s^{3}\right)^{2}}+\frac{\Gamma_{3}\left(y^{1}-y^{3}\right)}{\left(s^{2}\right)^{2}}\right), \\
& \frac{d y^{1}}{d t}=\frac{1}{2 \pi}\left(\frac{\Gamma_{2}\left(x^{1}-x^{2}\right)}{\left(s^{3}\right)^{2}}+\frac{\Gamma_{3}\left(x^{1}-x^{3}\right)}{\left(s^{2}\right)^{2}}\right), \\
& \frac{d x^{2}}{d t}=-\frac{1}{2 \pi}\left(\frac{\Gamma_{1}\left(y^{2}-y^{1}\right)}{\left(s^{3}\right)^{2}}+\frac{\Gamma_{3}\left(y^{2}-y^{3}\right)}{\left(s^{1}\right)^{2}}\right), \\
& \frac{d y^{2}}{d t}=\frac{1}{2 \pi}\left(\frac{\Gamma_{1}\left(x^{2}-x^{1}\right)}{\left(s^{3}\right)^{2}}+\frac{\Gamma_{3}\left(x^{2}-x^{3}\right)}{\left(s^{1}\right)^{2}}\right), \\
& \frac{d x^{3}}{d t}=-\frac{1}{2 \pi}\left(\frac{\Gamma_{1}\left(y^{3}-y^{1}\right)}{\left(s^{2}\right)^{2}}+\frac{\Gamma_{2}\left(y^{3}-y^{2}\right)}{\left(s^{1}\right)^{2}}\right), \\
& \frac{d y^{3}}{d t}=\frac{1}{2 \pi}\left(\frac{\Gamma_{1}\left(x^{3}-x^{1}\right)}{\left(s^{2}\right)^{2}}+\frac{\Gamma_{2}\left(x^{3}-x^{2}\right)}{\left(s^{1}\right)^{2}}\right) .
\end{aligned}
$$

Using Equations (11), (12), and (13), the motions of the three point vortices are shown on a two-dimensional plane. Further, to describe a three point vortex system as Hamilton's canonical form, the Hamiltonian of three point vortices is defined as follows:

$$
H=-\frac{1}{2 \pi}\left(\Gamma_{1} \Gamma_{2} \log s^{3}+\Gamma_{1} \Gamma_{3} \log s^{2}+\Gamma_{2} \Gamma_{3} \log s^{1}\right),
$$

where $\Gamma_{1}, \Gamma_{2}$, and $\Gamma_{3}$ are circulations of first, second, and third point vortices, respectively. Thus, from (11), (12), (13), and (14), equations of motions of point vortices are expressed as the following Hamilton's canonical form:

$$
\Gamma_{m} \frac{d x^{m}}{d t}=\frac{\partial H}{\partial y^{m}}, \quad \Gamma_{m} \frac{d y^{m}}{d t}=-\frac{\partial H}{\partial x^{m}},
$$

where $m=1,2,3$, and each left hand sides of Equation (15) are not summed. For all motions of point vortices, the Hamiltonian is constant. Then, Equation (15) is rewritten in terms of the relative distances $s^{1}, s^{2}$, and $s^{3}$ as follows:

$$
\begin{aligned}
& \frac{d\left(s^{1}\right)^{2}}{d t}=\frac{\Gamma_{1}}{2 \pi} \frac{J\left(s^{2}+s^{3}\right)\left(s^{2}-s^{3}\right)}{\left(s^{2} s^{3}\right)^{2}}, \\
& \frac{d\left(s^{2}\right)^{2}}{d t}=\frac{\Gamma_{2}}{2 \pi} \frac{J\left(s^{3}+s^{1}\right)\left(s^{3}-s^{1}\right)}{\left(s^{1} s^{3}\right)^{2}} \\
& \frac{d\left(s^{3}\right)^{2}}{d t}=\frac{\Gamma_{3}}{2 \pi} \frac{J\left(s^{1}+s^{2}\right)\left(s^{1}-s^{2}\right)}{\left(s^{1} s^{2}\right)^{2}}
\end{aligned}
$$

where $J$ is given by

$$
J^{2}=2\left(s^{2} s^{3}\right)^{2}+2\left(s^{3} s^{1}\right)^{2}+2\left(s^{1} s^{2}\right)^{2}-\left(s^{1}\right)^{4}-\left(s^{2}\right)^{4}-\left(s^{3}\right)^{4} .
$$

$J$ is an area of the triangle formed by the three point vortices. Moreover, Equations (16), (17), and (18) can be, respectively, rewritten as follows:

$$
\begin{gathered}
\frac{d s^{1}}{d t}=\frac{\Gamma_{1}}{4 \pi} \frac{J\left(s^{2}+s^{3}\right)\left(s^{2}-s^{3}\right)}{s^{1}\left(s^{2} s^{3}\right)^{2}}, \\
\frac{d s^{2}}{d t}=\frac{\Gamma_{2}}{4 \pi} \frac{J\left(s^{3}+s^{1}\right)\left(s^{3}-s^{1}\right)}{s^{2}\left(s^{1} s^{3}\right)^{2}}, \\
\frac{d s^{3}}{d t}=\frac{\Gamma_{3}}{4 \pi} \frac{J\left(s^{1}+s^{2}\right)\left(s^{1}-s^{2}\right)}{s^{3}\left(s^{1} s^{2}\right)^{2}} .
\end{gathered}
$$

Here, all initial values of $s^{1}, s^{2}$, and $s^{3}$ should be positive because $s^{1}, s^{2}$, and $s^{3}$ are relative distances. Subsequently, we use Equations (20), (21), and (22) to analyze geometric quantities of point vortices.

Next, we review the self-similarity of the three point vortices based on previous studies [30, 33, 35]. When the initial positions of the three point vortices are an equilateral triangle, all relative distances are constant since all right hand sides of Equations (20), (21), and (22) become zero; thus, the Hamiltonian (14) is constant even without circulation conditions. Then, the motions of point vortices are translation or rigid rotation. These two kinds of motions are classified by the circulation conditions. From [30], for translation, the circulations satisfy

$$
\Gamma_{1}+\Gamma_{2}+\Gamma_{3}=0
$$



isfies

Meanwhile, from [30], the motion of rigid rotation sat-

$$
\Gamma_{1}+\Gamma_{2}+\Gamma_{3} \neq 0
$$

When the initial positions of the three point vortices are not an equilateral triangle, all self-similar motions of the point vortex system satisfy

$$
\frac{1}{\Gamma_{1}}+\frac{1}{\Gamma_{2}}+\frac{1}{\Gamma_{3}}=0
$$

which is because the Hamiltonian has to be constant for a motion of point vortices. Moreover, the initial position of the third point vortex of all self-similar motions satisfies

$$
\begin{gathered}
x^{3}=\frac{\Gamma_{1} x^{1}+\Gamma_{2} x^{2}}{\Gamma_{1}+\Gamma_{2}}+\sqrt{\frac{\Gamma_{1}+\Gamma_{2}+\Gamma_{3}}{\Gamma_{1}+\Gamma_{2}}}\left|x^{1}-x^{2}\right| \cos \theta, \\
y^{3}=\sqrt{\frac{\Gamma_{1}+\Gamma_{2}+\Gamma_{3}}{\Gamma_{1}+\Gamma_{2}}}\left|x^{1}-x^{2}\right| \sin \theta,
\end{gathered}
$$

whereas the first and second point vortices lie on the $x$ -axis $\left(y^{1}=0, y^{2}=0\right)$. Here, $\theta$ is an angle between the $x$-axis and the line segment from the center of the circle to the position of the third point vortex (Figure 1), where the point of the center of circle is given by

$$
\left(x_{c}, y_{c}\right)=\left(\frac{\Gamma_{1} x^{1}+\Gamma_{2} x^{2}}{\Gamma_{1}+\Gamma_{2}}, 0\right) .
$$

Finally, we mention the linear stability of a motion of point vortices that satisfies the conditions (26) and (27) using relative distances and its Jacobian matrix. The equilibrium point is which all of left hand sides of Equations (20), (21), and (22) equal zero. Thus, for the equilibria, the relative distances satisfy the following:

$$
s^{1}=s^{2}=s^{3}=s,
$$

where $s$ is relative distances and $s>0$. From Equations (20), (21), and (22), the Jacobian matrix at the equilibrium point is given by

$$
A=\left[\begin{array}{ccc}
0 & \frac{\sqrt{3} \Gamma_{1}}{\pi(s)^{2}} & -\frac{\sqrt{3} \Gamma_{1}}{\pi(s)^{2}} \\
-\frac{\sqrt{3} \Gamma_{2}}{\pi(s)^{2}} & 0 & \frac{\sqrt{3} \Gamma_{2}}{\pi(s)^{2}} \\
\frac{\sqrt{3} \Gamma_{3}}{\pi(s)^{2}} & -\frac{\sqrt{3} \Gamma_{3}}{\pi(s)^{2}} & 0
\end{array}\right] .
$$

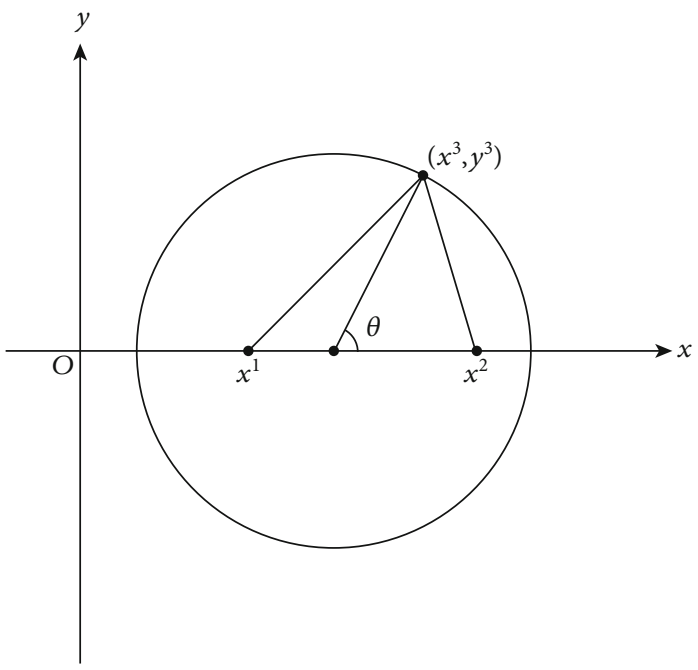

FIGURE 1: The circle of initial positions of self-similar expansion or collapse.

The eigenvalues of (30) is

$\left(\varepsilon_{1}, \varepsilon_{2}, \varepsilon_{3}\right)=\left(0,-\frac{\sqrt{-3\left(\Gamma_{1} \Gamma_{2}+\Gamma_{2} \Gamma_{3}+\Gamma_{3} \Gamma_{1}\right)}}{\pi(s)^{2}}, \frac{\sqrt{-3\left(\Gamma_{1} \Gamma_{2}+\Gamma_{2} \Gamma_{3}+\Gamma_{3} \Gamma_{1}\right)}}{\pi(s)^{2}}\right)$,

where $\varepsilon_{1}, \varepsilon_{2}$, and $\varepsilon_{3}$ are the first, second, and third eigenvalues of the matrix (30). From (31), since $\varepsilon_{1}=0$, the linear stability around the equilibrium point of the three point vortex system is not asymptotically stable but is Lyapunov stable when $\Gamma_{1} \Gamma_{2}+\Gamma_{2} \Gamma_{3}+\Gamma_{3} \Gamma_{1}>0$.

In this article, we handle expansion and collapse as the self-similar motion of point vortices.

\section{Relationship between Three Point Vortices and a Deviation Curvature}

In this section, a motion of three point vortices is discussed from the viewpoint of differential geometry. The KCC theory is applied to the point vortex system, and the deviation curvature is obtained. Then, the expansion and collapse of point vortices are classified by the deviation curvature for both selfand nonself-similar motions.

4.1. Eigenvalues of Deviation Curvature of a Three Point Vortex System. First, we calculate eigenvalues of deviation curvatures in a three point vortex system that exhibits general motions including self- and nonself-similarity. For applying the KCC theory, Equations (20), (21), and (22) are differentiated by time parameter $t$ as follows:

$$
\begin{aligned}
\ddot{s}^{1}= & \frac{\Gamma_{1} J}{4 \pi}\left\{\frac{d(\log J)}{d t} \frac{\left(s^{2}+s^{3}\right)\left(s^{2}-s^{3}\right)}{s^{1}\left(s^{2} s^{3}\right)^{2}}\right. \\
& \left.+\frac{2 s^{1} s^{2} s^{3}\left(s^{2} \dot{s}^{2}-s^{3} \dot{s}^{3}\right)-\left(s^{2}+s^{3}\right)\left(s^{2}-s^{3}\right)\left(\dot{s}^{1} s^{2} s^{3}+2 s^{1} \dot{s}^{2} s^{3}+2 s^{1} s^{2} \dot{s}^{3}\right)}{\left(s^{1}\right)^{2}\left(s^{2} s^{3}\right)^{3}}\right\},
\end{aligned}
$$




$$
\begin{aligned}
\ddot{s}^{2}= & \frac{\Gamma_{2} J}{4 \pi}\left\{\frac{d(\log J)}{d t} \frac{\left(s^{3}+s^{1}\right)\left(s^{3}-s^{1}\right)}{s^{2}\left(s^{1} s^{3}\right)^{2}}\right. \\
& \left.+\frac{2 s^{1} s^{2} s^{3}\left(s^{3} \dot{s}^{3}-s^{1} \dot{s}^{1}\right)-\left(s^{3}+s^{1}\right)\left(s^{3}-s^{1}\right)\left(\dot{s}^{2} s^{1} s^{3}+2 s^{2} \dot{s}^{1} s^{3}+2 s^{2} s^{1} \dot{s}^{3}\right)}{\left(s^{2}\right)^{2}\left(s^{1} s^{3}\right)^{3}}\right\},
\end{aligned}
$$

$$
\begin{aligned}
\ddot{s}^{3}= & \frac{\Gamma_{3} J}{4 \pi}\left\{\frac{d(\log J)}{d t} \frac{\left(s^{1}+s^{2}\right)\left(s^{1}-s^{2}\right)}{s^{3}\left(s^{1} s^{2}\right)^{2}}\right. \\
& \left.+\frac{2 s^{1} s^{2} s^{3}\left(s^{1} \dot{s}^{1}-s^{2} \dot{s}^{2}\right)-\left(s^{1}+s^{2}\right)\left(s^{1}-s^{2}\right)\left(\dot{s}^{3} s^{1} s^{2}+2 s^{3} \dot{s}^{1} s^{2}+2 s^{3} s^{1} \dot{s}^{2}\right)}{\left(s^{3}\right)^{2}\left(s^{1} s^{2}\right)^{3}}\right\} .
\end{aligned}
$$

Here, the initial values $s^{i}(0)$ of Equations (32), (33), and (34) have to be positive, and $\dot{s}^{i}(0)$ are determined by Equations (20), (21), and (22). From Equations (32), (33), and (34), the function $G^{i}$, nonlinear connection $N_{j}^{\mathrm{i}}$ and Berwald connection $G_{j k}^{i}$ can be obtained (Appendix A). Then, a deviation curvature of the three point vortex system becomes a $3 \times 3$ matrix $P_{j}^{i}$, and the characteristic equation is written as follows:

$$
\lambda^{3}-\operatorname{tr} P_{j}^{i} \lambda^{2}+\frac{1}{2}\left[\left(\operatorname{tr} P_{j}^{i}\right)^{2}-\operatorname{tr}\left\{\left(P_{j}^{i}\right)^{2}\right\}\right] \lambda-\operatorname{det} P_{j}^{i}=0,
$$

where $\lambda$ is an eigenvalue of $P_{j}^{i}$. Let $\lambda_{1}, \lambda_{2}, \lambda_{3}$, and $\lambda_{n}$ be the first, second, third, and $n$th eigenvalues of a deviation curvature. From the characteristic equation (35) and Cardano's formula of a cubic equation [39], the $n$th eigenvalue of the deviation curvature of a general motion system including self- and nonself-similarity is given by

$$
\lambda_{n}=\alpha \sqrt[3]{-\frac{q}{2}+\sqrt{\left(\frac{q}{2}\right)^{2}+\left(\frac{p}{3}\right)^{3}}}+\beta \sqrt[3]{-\frac{q}{2}-\sqrt{\left(\frac{q}{2}\right)^{2}+\left(\frac{p}{3}\right)^{3}}}+\frac{1}{3} \operatorname{tr} P_{j}^{i}
$$

where

$$
\begin{gathered}
(n, \alpha, \beta)=(1,1,1),\left(2, \frac{-1+\sqrt{3} i}{2}, \frac{-1-\sqrt{3} i}{2}\right),\left(3, \frac{-1-\sqrt{3} i}{2}, \frac{-1+\sqrt{3} i}{2}\right), \\
p=-\frac{1}{3}\left(\operatorname{tr} P_{j}^{i}\right)^{2}+\frac{1}{2}\left[\left(\operatorname{tr} P_{j}^{i}\right)^{2}-\operatorname{tr}\left\{\left(P_{j}^{i}\right)^{2}\right\}\right], \\
q=-\frac{2}{27}\left(\operatorname{tr} P_{j}^{i}\right)^{3}+\frac{1}{6} \operatorname{tr} P_{j}^{i}\left[\left(\operatorname{tr} P_{j}^{i}\right)^{2}-\operatorname{tr}\left\{\left(P_{j}^{i}\right)^{2}\right\}\right]-\operatorname{det} P_{j}^{i} .
\end{gathered}
$$

The condition in which the eigenvalues (36) become double or triple repeated roots is given by discriminant $D$ :

$$
D=\left(\frac{q}{2}\right)^{2}+\left(\frac{p}{3}\right)^{3}
$$

When $D=0$, the eigenvalues (36) become double or triple repeated roots. For $D=0$ and $q=0$, the eigenvalues (36) become triple repeated roots.

4.2. Classification of Self-Similar Motions of a Three Point Vortex System by Deviation Curvature. In this section, we consider a motion of self-similarity of point vortices based on $[30,33,35]$ :

$$
s^{1}(t)=f(t) C_{1}, s^{2}(t)=f(t) C_{2}, s^{3}(t)=f(t) C_{3},
$$

where $C_{1}, C_{2}$, and $C_{3}$ are constants that are determined by the initial positions of point vortices. From [33], the particular form of the function $f(t)$ is given by

$$
f(t)=\sqrt{1-\frac{t}{\tau}}
$$

where $\tau$ is the collapse time if $\tau>0$, and if $\tau<0$, the function $f(t)$ increases monotonically.

For simplicity, using (25), let $\Gamma_{3}$ be as follows:

$$
\Gamma_{3}=-\frac{\Gamma_{1} \Gamma_{2}}{\Gamma_{1}+\Gamma_{2}} .
$$
lows:

Then, $\operatorname{tr} P_{j}^{i}, \operatorname{tr}\left\{\left(P_{j}^{i}\right)^{2}\right\}$, and det $P_{j}^{i}$ can be expressed as fol-

$$
\begin{gathered}
\operatorname{tr} P_{j}^{i}=\frac{u\left(s^{1}, s^{2}, s^{3}\right)}{(\pi J)^{2}\left(\Gamma_{1}+\Gamma_{2}\right)^{2}\left(2 s^{1} s^{2} s^{3}\right)^{4}}, \\
\operatorname{tr}\left\{\left(P_{j}^{i}\right)^{2}\right\}=\frac{v\left(s^{1}, s^{2}, s^{3}\right)}{(\pi J)^{4}\left(\Gamma_{1}+\Gamma_{2}\right)^{4}\left(2 s^{1} s^{2} s^{3}\right)^{8}} \\
\operatorname{det} P_{j}^{i}=-\frac{w\left(s^{1}, s^{2}, s^{3}\right)}{\pi^{6}\left(2 s^{1} s^{2} s^{3}\right)^{12}},
\end{gathered}
$$

where the details of (46) are described in Appendix B.

Using (41) and (46), $\operatorname{tr} P_{j}^{i}, \operatorname{tr}\left\{\left(P_{j}^{i}\right)^{2}\right\}$, and $\operatorname{det} P_{j}^{i}$ can be rewritten as follows:

$$
\begin{gathered}
\operatorname{tr} P_{j}^{i}=\frac{C_{\mathrm{tr}}}{f(t)^{4}}, \\
\operatorname{tr}\left\{\left(P_{j}^{i}\right)^{2}\right\}=\frac{C_{\mathrm{tr} 2}}{f(t)^{8}}, \\
\operatorname{det} P_{j}^{i}=\frac{C_{\mathrm{det}}}{f(t)^{12}},
\end{gathered}
$$

where $C_{\mathrm{tr}}, C_{\mathrm{tr} 2}$, and $C_{\mathrm{det}}$ are determined by $\Gamma_{1}, \Gamma_{2}, C_{1}, C_{2}$, and $C_{3}$. Therefore, from (36) and (47), eigenvalues of a deviation curvature of self-similar motions can be rewritten as follows:

$$
\lambda_{n}=\frac{1}{f(t)^{4}}\left(\alpha C^{\prime}+\beta C^{\prime \prime}+C^{\prime \prime \prime}\right)
$$




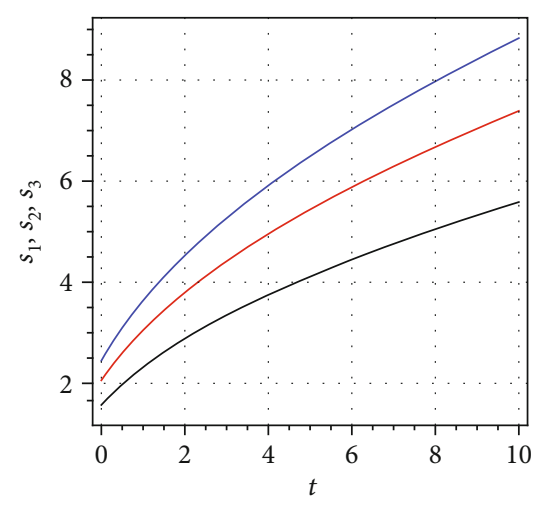

(a)

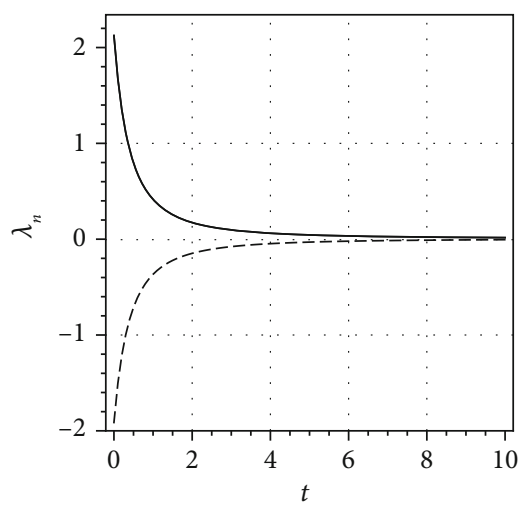

(b)

Figure 2: (a) The time evolutions of relative distances $s^{1}, s^{2}$, and $s^{3}$. (b) The time evolutions of the $n$th eigenvalue of the deviation curvature in a self-similar expansion.

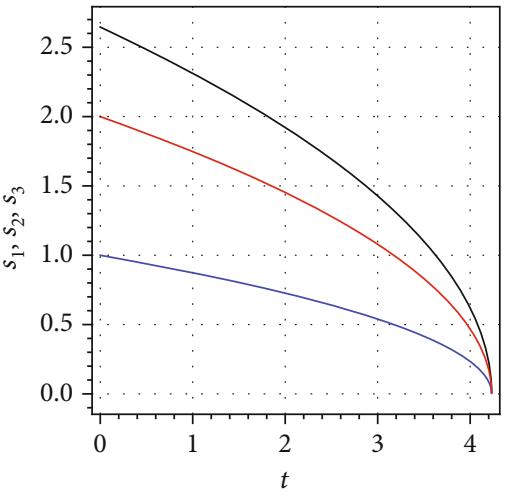

(a)

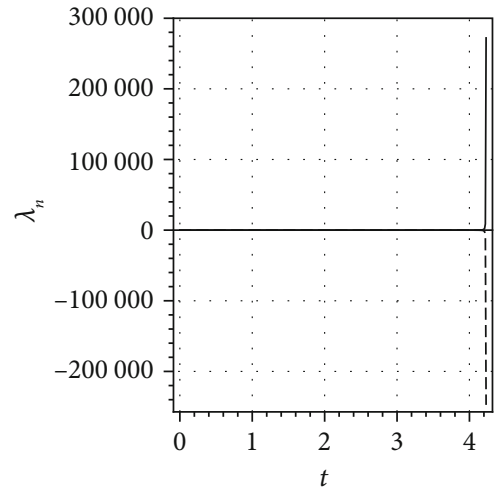

(b)

FIGURE 3: (a) The time evolutions of relative distances $s^{1}, s^{2}$, and $s^{3}$. (b) The time evolutions of the $n$th eigenvalue of the deviation curvature in a self-similar collapse.

where $C^{\prime}, C^{\prime \prime}$, and $C^{\prime \prime \prime}$ are determined by $\Gamma_{1}, \Gamma_{2}, C_{1}, C_{2}$, and $C_{3}$; thus, the term $\alpha C^{\prime}+\beta C^{\prime \prime}+C^{\prime \prime \prime}$ is constant that means independent of the time $t$. From (48), eigenvalues of self-similarity are proportional to the inverse fourth power of relative distances. Accordingly, since $f(t)$ of (42) is monotonous, for expansion, the eigenvalues of the deviation curvature monotonically converge to zero, whereas for collapse, the eigenvalues of the deviation curvature monotonically diverge. Thus, when the strengths of interactions between vortices decrease, the relative distances are increase, that is expansion, and eigenvalues of the deviation curvature monotonically converge to zero from (48). When the strengths of interactions between vortices increase, relative distances decrease, that is collapse, and eigenvalues of the deviation curvature monotonically diverge from (48). These indicate that the strengths of interactions between vortices are related to the time evolution of spatial geometric structure in terms of the deviation curvature. In particular, for collapse, the collision point becomes a geometric singularity because the eigenvalues of the deviation curvature are divergence. These results mean that motions of self-similarity are classified by the eigenvalues of the deviation curvature.
Next, we consider specific examples of expansion and collapse based on the discriminant $D$. When the third point vortex is on the circle of Figure 1, using $\left(x^{1}, y^{1}\right)=(-1,0)$, ( $\left.x^{2}, y^{2}\right)=(1,0)$ and $\left(\Gamma_{1}, \Gamma_{2}, \Gamma_{3}\right)=(2 \Gamma, 2 \Gamma,-\Gamma)$, the discriminant (40) is zero $(D=0)$, and $q$ of (39) is given by

$$
q=\frac{91125 \Gamma \sin ^{6} 2 \theta}{108 \pi^{6}(5-3 \cos 2 \theta)^{6}} .
$$

According to [30,33,35], when $\theta=0, \pi / 2, \pi$, and $3 \pi / 2$, the motions of point vortices are not expansion and collapse. This means $q \neq 0$ for expansion and collapse. Therefore, for expansion and collapse, the eigenvalues (36) always have double repeated roots. This result suggests that eigenvalues of a deviation curvature are affected by the assumption of self-similarity, which implies a decrease in degrees of freedom. The results for expansion and collapse can be confirmed numerically. Initial conditions and circulations for the expansion are given by

$$
\left(\Gamma_{1}, \Gamma_{2}, \Gamma_{3}\right)=(40,40,-20),
$$




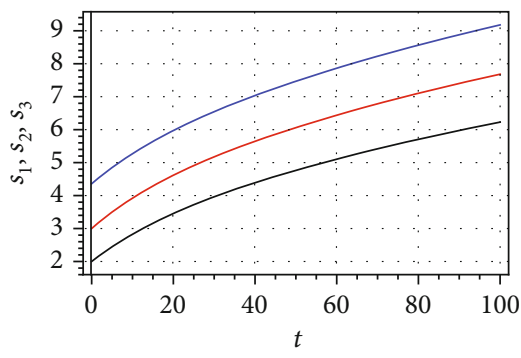

(a)

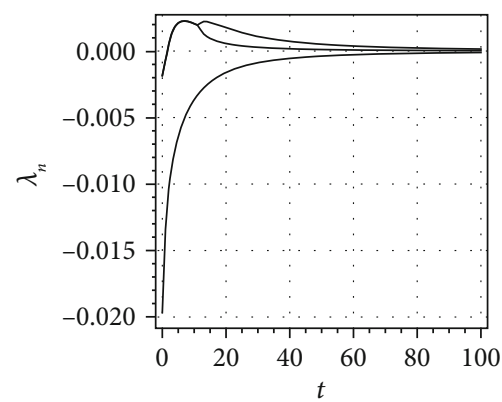

(b)

Figure 4: (a) The time evolutions of relative distances $s^{1}, s^{2}$, and $s^{3}$. (b) The time evolutions of the $n$th eigenvalue of the deviation curvature in a nonself-similar expansion.

$$
\begin{gathered}
\left(x^{1}(0), y^{1}(0)\right)=(-1,0), \\
\left(x^{2}(0), y^{2}(0)\right)=(1,0), \\
\left(x^{3}(0), y^{3}(0)\right)=\left(\frac{\sqrt{3}}{2},-\frac{3}{2}\right), \\
\left(s^{1}(0), s^{2}(0), s^{3}(0)\right)=\left(\sqrt{\left(1-\frac{\sqrt{3}}{2}\right)^{2}+\frac{9}{4}}, \sqrt{\left(1+\frac{\sqrt{3}}{2}\right)^{2}+\frac{9}{4}}, 2\right),
\end{gathered}
$$

where expression (53) satisfies conditions (26) and (27), and $\theta=-\pi / 3$. Moreover, initial position and circulation conditions for the collapse are given by

$$
\begin{gathered}
\left(\Gamma_{1}, \Gamma_{2}, \Gamma_{3}\right)=(4,4,-2), \\
\left(x^{1}(0), y^{1}(0)\right)=(-1,0), \\
\left(x^{2}(0), y^{2}(0)\right)=(1,0), \\
\left(x^{3}(0), y^{3}(0)\right)=\left(-\frac{3}{2},-\frac{\sqrt{3}}{2}\right), \\
\left(s^{1}(0), s^{2}(0), s^{3}(0)\right)=(\sqrt{7}, 1,2),
\end{gathered}
$$

where (58) satisfies conditions (26) and (27), and $\theta=-5 \pi / 6$. Then, variations in relative distances and time evolutions of the deviation curvature are shown in Figures 2 and 3. The relative distances $s^{1}, s^{2}$, and $s^{3}$ are represented by black, blue, and red, respectively. For the eigenvalues of the deviation curvature, double repeated roots are represented by a solid line, and the other roots are represented by a dashed line. For expansion in Figure 2, the eigenvalues of the deviation curvature monotonically converge to zero, whereas for collapse in Figure 3, the eigenvalues of the deviation curvature monotonically diverge. The other KCC invariants are shown in Appendix C.

4.3. Numerical Example of a Nonself-Similar Motion. In this section, the variations in relative distances and time evolutions of eigenvalues of the deviation curvature are analyzed numerically for a nonself-similar expansion. The initial positions and circulations are given by

$$
\begin{gathered}
\left(\Gamma_{1}, \Gamma_{2}, \Gamma_{3}\right)=(2,2,-1), \\
\left(s^{1}(0), s^{2}(0), s^{3}(0)\right)=(2, \sqrt{19}, 3) .
\end{gathered}
$$

The initial position (61) does not satisfy the self-similar condition shown in $[30,33,35]$. Figure 4 shows the variation of relative distances and time evolutions of eigenvalues of the deviation curvature. The relative distances $s^{1}, s^{2}$, and $s^{3}$ are represented by black, blue, and red, respectively. In this case, the eigenvalues of the deviation curvature are nonmonotonous but converge to zero. This result suggests that the motions of a nonself-similarity system of three point vortices can be classified by eigenvalues of the deviation curvature as well as motions of a self-similarity system.

\section{Conclusions}

The KCC theory has been applied to a three point vortex system, and eigenvalues of a deviation curvature have been calculated using relative distances. For self-similar motions, the eigenvalues of deviation curvature are proportional to the inverse fourth power of relative distances. We consider the classification of self-similar motion of point vortices by eigenvalues of the deviation curvature. The details of the classification are as follows. For expansion, the eigenvalues are double repeated roots, and the eigenvalues of the deviation curvature monotonically converge to zero. For collapse, the eigenvalues are double repeated roots, and the eigenvalues of the deviation curvature monotonically diverge. These results mean that self-similar motions are classified by eigenvalues of the deviation curvature. Further, in the case of nonself-similar expansion, eigenvalues of the deviation curvature are not monotonous but convergent to zero. This numerical result suggests that motions of a nonself-similar system of three point vortices can be classified by eigenvalues of a deviation curvature as well as motions of a self-similarity system. 


\section{Appendix}

\section{A. Details of Nonlinear Connection and Berwald Connection}

In this section, we show the details of the function $G^{i}$, nonlinear connection $N_{j}^{i}$, and Berwald connection $G_{j k}^{i}$ mentioned in Section 4. From Equation (1), $G^{i}$ is rewritten as $G^{i}=-\ddot{s}^{i} / 2$ :

$$
\begin{aligned}
G^{1}= & -\frac{\Gamma_{1} J}{4 \pi}\left\{\frac{d(\log J)}{d t} \frac{\left(s^{2}+s^{3}\right)\left(s^{2}-s^{3}\right)}{s^{1}\left(s^{2} s^{3}\right)^{2}}\right. \\
& \left.+\frac{2 s^{1} s^{2} s^{3}\left(s^{2} \dot{s}^{2}-s^{3} \dot{s}^{3}\right)-\left(s^{2}+s^{3}\right)\left(s^{2}-s^{3}\right)\left(\dot{s}^{1} s^{2} s^{3}+2 s^{1} \dot{s}^{2} s^{3}+2 s^{1} s^{2} \dot{s}^{3}\right)}{\left(s^{1}\right)^{2}\left(s^{2} s^{3}\right)^{3}}\right\},
\end{aligned}
$$

$$
\begin{aligned}
G^{2}= & -\frac{\Gamma_{2} J}{4 \pi}\left\{\frac{d(\log J)}{d t} \frac{\left(s^{3}+s^{1}\right)\left(s^{3}-s^{1}\right)}{s^{2}\left(s^{1} s^{3}\right)^{2}}\right. \\
& \left.+\frac{2 s^{1} s^{2} s^{3}\left(s^{3} \dot{s}^{3}-s^{1} \dot{s}^{1}\right)-\left(s^{3}+s^{1}\right)\left(s^{3}-s^{1}\right)\left(\dot{s}^{2} s^{1} s^{3}+2 s^{2} \dot{s}^{1} s^{3}+2 s^{2} s^{1} \dot{s}^{3}\right)}{\left(s^{2}\right)^{2}\left(s^{1} s^{3}\right)^{3}}\right\},
\end{aligned}
$$

$$
\begin{aligned}
G^{3}= & -\frac{\Gamma_{3} J}{4 \pi}\left\{\frac{d(\log J)}{d t} \frac{\left(s^{1}+s^{2}\right)\left(s^{1}-s^{2}\right)}{s^{3}\left(s^{1} s^{2}\right)^{2}}\right. \\
& \left.+\frac{2 s^{1} s^{2} s^{3}\left(s^{1} \dot{s}^{1}-s^{2} \dot{s}^{2}\right)-\left(s^{1}+s^{2}\right)\left(s^{1}-s^{2}\right)\left(\dot{s}^{3} s^{1} s^{2}+2 s^{3} \dot{s}^{1} s^{2}+2 s^{3} s^{1} \dot{s}^{2}\right)}{\left(s^{3}\right)^{2}\left(s^{1} s^{2}\right)^{3}}\right\} .
\end{aligned}
$$

From expressions (A.1), (A.2), and (A.3), coefficients of nonlinear connection $N_{j}^{i}$ are given by

$$
\begin{gathered}
N_{1}^{1}=-\frac{\Gamma_{1}\left(s^{2}+s^{3}\right)\left(s^{2}-s^{3}\right)}{4 \pi s^{1}\left(s^{2} s^{3}\right)^{2}}\left(\frac{\partial j}{\partial \dot{s}^{1}}-\frac{J}{s^{1}}\right), \\
N_{2}^{1}=-\frac{\Gamma_{1}}{4 \pi s^{1}\left(s^{2} s^{3}\right)^{2}}\left(\frac{\partial j}{\partial \dot{s}^{2}}\left(s^{2}+s^{3}\right)\left(s^{2}-s^{3}\right)+\frac{2 J}{s^{2}}\left(s^{3}\right)^{2}\right), \\
N_{3}^{1}=-\frac{\Gamma_{1}}{4 \pi s^{1}\left(s^{2} s^{3}\right)^{2}}\left(\frac{\partial j}{\partial \dot{s}^{3}}\left(s^{2}+s^{3}\right)\left(s^{2}-s^{3}\right)-\frac{2 J}{s^{3}}\left(s^{2}\right)^{2}\right), \\
N_{1}^{2}=-\frac{\Gamma_{2}}{4 \pi s^{2}\left(s^{1} s^{3}\right)^{2}}\left(\frac{\partial j}{\partial \dot{s}^{1}}\left(s^{3}+s^{1}\right)\left(s^{3}-s^{1}\right)-\frac{2 J}{s^{1}}\left(s^{3}\right)^{2}\right), \\
N_{2}^{2}=-\frac{\Gamma_{2}\left(s^{3}+s^{1}\right)\left(s^{3}-s^{1}\right)}{4 \pi s^{2}\left(s^{1} s^{3}\right)^{2}}\left(\frac{\partial j}{\partial \dot{s}^{2}}-\frac{J}{s^{2}}\right), \\
N_{3}^{2}=-\frac{\Gamma_{2}}{4 \pi s^{2}\left(s^{1} s^{3}\right)^{2}}\left(\frac{\partial j}{\partial \dot{s}^{3}}\left(s^{3}+s^{1}\right)\left(s^{3}-s^{1}\right)+\frac{2 J}{s^{3}}\left(s^{1}\right)^{2}\right),
\end{gathered}
$$

$$
N_{1}^{3}=-\frac{\Gamma_{3}}{4 \pi s^{3}\left(s^{1} s^{2}\right)^{2}}\left(\frac{\partial \dot{J}}{\partial \dot{s}^{1}}\left(s^{1}+s^{2}\right)\left(s^{1}-s^{2}\right)+\frac{2 J}{s^{1}}\left(s^{2}\right)^{2}\right),
$$

$$
N_{2}^{3}=-\frac{\Gamma_{3}}{4 \pi s^{3}\left(s^{1} s^{2}\right)^{2}}\left(\frac{\partial \dot{J}}{\partial \dot{s}^{2}}\left(s^{1}+s^{2}\right)\left(s^{1}-s^{2}\right)-\frac{2 J}{s^{2}}\left(s^{1}\right)^{2}\right) \text {, }
$$

$$
N_{3}^{3}=-\frac{\Gamma_{3}\left(s^{1}+s^{2}\right)\left(s^{1}-s^{2}\right)}{4 \pi s^{3}\left(s^{1} s^{2}\right)^{2}}\left(\frac{\partial j}{\partial \dot{s}^{3}}-\frac{J}{s^{3}}\right),
$$

where

$$
\begin{gathered}
\dot{J}=\frac{2}{J}\left\{s^{2} \dot{s}^{2}\left(s^{3}\right)^{2}+\left(s^{2}\right)^{2} s^{3} \dot{s}^{3}+s^{3} \dot{s}^{3}\left(s^{1}\right)^{2}+\left(s^{3}\right)^{2} s^{1} \dot{s}^{1}\right. \\
\left.+s^{1} \dot{s}^{1}\left(s^{2}\right)^{2}+\left(s^{1}\right)^{2} s^{2} \dot{s}^{2}-\left(s^{1}\right)^{3} \dot{s}^{1}-\left(s^{2}\right)^{3} \dot{s}^{2}-\left(s^{3}\right)^{3} \dot{s}^{3}\right\} \\
\frac{\partial \dot{J}}{\partial \dot{s}^{1}}=\frac{2}{J} s^{1}\left\{\left(s^{2}\right)^{2}+\left(s^{3}\right)^{2}-\left(s^{1}\right)^{2}\right\} \\
\frac{\partial \dot{J}}{\partial \dot{s}^{2}}=\frac{2}{J} s^{2}\left\{\left(s^{3}\right)^{2}+\left(s^{1}\right)^{2}-\left(s^{2}\right)^{2}\right\} \\
\frac{\partial \dot{j}}{\partial \dot{s}^{3}}=\frac{2}{J} s^{3}\left\{\left(s^{1}\right)^{2}+\left(s^{2}\right)^{2}-\left(s^{3}\right)^{2}\right\}
\end{gathered}
$$

Then, from expressions (A.4)-(A.12), $\dot{s}^{1}, \dot{s}^{2}$, and $\dot{s}^{3}$ are not included in $N_{j}^{i}$, therefore $G_{j k}^{i}=0$.

\section{B. Details of Trace and Determinant of Deviation Curvature}

In this section, we describe in detail $\operatorname{tr} P_{j}^{i}, \operatorname{tr}\left\{\left(P_{j}^{i}\right)^{2}\right\}$, and det $P_{j}^{i}$ on the assumptions of self-similarity and (43) mentioned in Section $4, \operatorname{tr} P_{j}^{i}, \operatorname{tr}\left\{\left(P_{j}^{i}\right)^{2}\right\}$, and $\operatorname{det} P_{j}^{i}$ are given by

$$
\begin{gathered}
\operatorname{tr} P_{j}^{i}=\frac{u\left(s^{1}, s^{2}, s^{3}\right)}{(\pi J)^{2}\left(\Gamma_{1}+\Gamma_{2}\right)^{2}\left(2 s^{1} s^{2} s^{3}\right)^{4}}, \\
\operatorname{tr}\left\{\left(P_{j}^{i}\right)^{2}\right\}=\frac{v\left(s^{1}, s^{2}, s^{3}\right)}{(\pi J)^{4}\left(\Gamma_{1}+\Gamma_{2}\right)^{4}\left(2 s^{1} s^{2} s^{3}\right)^{8}}, \\
\operatorname{det} P_{j}^{i}=-\frac{w\left(s^{1}, s^{2}, s^{3}\right)}{\pi^{6}\left(2 s^{1} s^{2} s^{3}\right)^{12}},
\end{gathered}
$$

where

$u\left(s^{1}, s^{2}, s^{3}\right)=3\left(\Gamma_{2}\right)^{2}\left(30\left(\Gamma_{1}\right)^{2}+30\left(\Gamma_{2}\right)\left(\Gamma_{1}\right)+7\left(\Gamma_{2}\right)^{2}\right)\left(s^{1}\right)^{12}-2\left(\Gamma_{2}\right)\left(\left(-34\left(\Gamma_{1}\right)^{3}+121\left(\Gamma_{2}\right)\left(\Gamma_{1}\right)^{2}\right.\right.$ $\left.+122\left(\Gamma_{2}\right)^{2}\left(\Gamma_{1}\right)+30\left(\Gamma_{2}\right)^{3}\right)\left(s^{2}\right)^{2}+\left(34\left(\Gamma_{1}\right)^{3}+223\left(\Gamma_{2}\right)\left(\Gamma_{1}\right)^{2}+222\left(\Gamma_{2}\right)^{2}\left(\Gamma_{1}\right)\right.$ $\left.\left.+63\left(\Gamma_{2}\right)^{3}\right)\left(s^{3}\right)^{2}\right)\left(s^{1}\right)^{10}+\left(\left(\left(\Gamma_{1}\right)^{4}-214\left(\Gamma_{2}\right)\left(\Gamma_{1}\right)^{3}+182\left(\Gamma_{2}\right)^{2}\left(\Gamma_{1}\right)^{2}+140\left(\Gamma_{2}\right)^{3}\left(\Gamma_{1}\right)\right.\right.$ $\left.+58\left(\Gamma_{2}\right)^{4}\right)\left(s^{2}\right)^{4}-2\left(\left(\Gamma_{1}\right)^{4}+2\left(\Gamma_{2}\right)\left(\Gamma_{1}\right)^{3}-229\left(\Gamma_{2}\right)^{2}\left(\Gamma_{1}\right)^{2}-230\left(\Gamma_{2}\right)^{3}\left(\Gamma_{1}\right)\right.$ 
$\left.-90\left(\Gamma_{2}\right)^{4}\right)\left(s^{3}\right)^{2}\left(s^{2}\right)^{2}+\left(\left(\Gamma_{1}\right)^{4}+218\left(\Gamma_{2}\right)\left(\Gamma_{1}\right)^{3}+830\left(\Gamma_{2}\right)^{2}\left(\Gamma_{1}\right)^{2}+870\left(\Gamma_{2}\right)^{3}\left(\Gamma_{1}\right)\right.$ $\left.\left.+315\left(\Gamma_{2}\right)^{4}\right)\left(s^{3}\right)^{4}\right)\left(s^{1}\right)^{8}-4\left(5\left(\left(\Gamma_{1}\right)^{4}-8\left(\Gamma_{2}\right)\left(\Gamma_{1}\right)^{3}+3\left(\Gamma_{2}\right)^{2}\left(\Gamma_{1}\right)^{2}-8\left(\Gamma_{2}\right)^{3}\left(\Gamma_{1}\right)\right.\right.$ $\left.+\left(\Gamma_{2}\right)^{4}\right)\left(s^{2}\right)^{6}+\left(-5\left(\Gamma_{1}\right)^{4}+50\left(\Gamma_{2}\right)\left(\Gamma_{1}\right)^{3}+123\left(\Gamma_{2}\right)^{2}\left(\Gamma_{1}\right)^{2}+96\left(\Gamma_{2}\right)^{3}\left(\Gamma_{1}\right)\right.$ $\left.+58\left(\Gamma_{2}\right)^{4}\right)\left(s^{3}\right)^{2}\left(s^{2}\right)^{4}-\left(5\left(\Gamma_{1}\right)^{4}+70\left(\Gamma_{2}\right)\left(\Gamma_{1}\right)^{3}+57\left(\Gamma_{2}\right)^{2}\left(\Gamma_{1}\right)^{2}+20\left(\Gamma_{2}\right)^{3}\left(\Gamma_{1}\right)\right.$ $\left.-30\left(\Gamma_{2}\right)^{4}\right)\left(s^{3}\right)^{4}\left(s^{2}\right)^{2}+5\left(\left(\Gamma_{1}\right)^{4}+12\left(\Gamma_{2}\right)\left(\Gamma_{1}\right)^{3}+33\left(\Gamma_{2}\right)^{2}\left(\Gamma_{1}\right)^{2}+42\left(\Gamma_{2}\right)^{3}\left(\Gamma_{1}\right)\right.$ $\left.\left.+21\left(\Gamma_{2}\right)^{4}\right)\left(s^{3}\right)^{6}\right)\left(s^{1}\right)^{6}+\left(\left(58\left(\Gamma_{1}\right)^{4}+140\left(\Gamma_{2}\right)\left(\Gamma_{1}\right)^{3}+182\left(\Gamma_{2}\right)^{2}\left(\Gamma_{1}\right)^{2}\right.\right.$ $\left.-214\left(\Gamma_{2}\right)^{3}\left(\Gamma_{1}\right)+\left(\Gamma_{2}\right)^{4}\right)\left(s^{2}\right)^{8}-4\left(58\left(\Gamma_{1}\right)^{4}+96\left(\Gamma_{2}\right)\left(\Gamma_{1}\right)^{3}+123\left(\Gamma_{2}\right)^{2}\left(\Gamma_{1}\right)^{2}\right.$ $\left.+50\left(\Gamma_{2}\right)^{3}\left(\Gamma_{1}\right)-5\left(\Gamma_{2}\right)^{4}\right)\left(s^{3}\right)^{2}\left(s^{2}\right)^{6}+348\left(\left(\Gamma_{1}\right)^{2}+\left(\Gamma_{2}\right)\left(\Gamma_{1}\right)+\left(\Gamma_{2}\right)^{2}\right)^{2}\left(s^{3}\right)^{4}\left(s^{2}\right)^{4}$ $-4\left(58\left(\Gamma_{1}\right)^{4}+136\left(\Gamma_{2}\right)\left(\Gamma_{1}\right)^{3}+183\left(\Gamma_{2}\right)^{2}\left(\Gamma_{1}\right)^{2}+140\left(\Gamma_{2}\right)^{3}\left(\Gamma_{1}\right)+30\left(\Gamma_{2}\right)^{4}\right)\left(s^{3}\right)^{6}\left(s^{2}\right)^{2}$ $\left.+\left(58\left(\Gamma_{1}\right)^{4}+92\left(\Gamma_{2}\right)\left(\Gamma_{1}\right)^{3}+110\left(\Gamma_{2}\right)^{2}\left(\Gamma_{1}\right)^{2}+390\left(\Gamma_{2}\right)^{3}\left(\Gamma_{1}\right)+315\left(\Gamma_{2}\right)^{4}\right)\left(s^{3}\right)^{8}\right)\left(s^{1}\right)^{4}$ $-2\left(\left(\Gamma_{1}\right)\left(30\left(\Gamma_{1}\right)^{3}+122\left(\Gamma_{2}\right)\left(\Gamma_{1}\right)^{2}+121\left(\Gamma_{2}\right)^{2}\left(\Gamma_{1}\right)-34\left(\Gamma_{2}\right)^{3}\right)\left(s^{2}\right)^{10}+\left(-90\left(\Gamma_{1}\right)^{4}\right.\right.$ $\left.-230\left(\Gamma_{2}\right)\left(\Gamma_{1}\right)^{3}-229\left(\Gamma_{2}\right)^{2}\left(\Gamma_{1}\right)^{2}+2\left(\Gamma_{2}\right)^{3}\left(\Gamma_{1}\right)+\left(\Gamma_{2}\right)^{4}\right)\left(s^{3}\right)^{2}\left(s^{2}\right)^{8}+2\left(30\left(\Gamma_{1}\right)^{4}\right.$ $\left.-20\left(\Gamma_{2}\right)\left(\Gamma_{1}\right)^{3}-57\left(\Gamma_{2}\right)^{2}\left(\Gamma_{1}\right)^{2}-70\left(\Gamma_{2}\right)^{3}\left(\Gamma_{1}\right)-5\left(\Gamma_{2}\right)^{4}\right)\left(s^{3}\right)^{4}\left(s^{2}\right)^{6}+2\left(30\left(\Gamma_{1}\right)^{4}\right.$ $\left.+140\left(\Gamma_{2}\right)\left(\Gamma_{1}\right)^{3}+183\left(\Gamma_{2}\right)^{2}\left(\Gamma_{1}\right)^{2}+136\left(\Gamma_{2}\right)^{3}\left(\Gamma_{1}\right)+58\left(\Gamma_{2}\right)^{4}\right)\left(s^{3}\right)^{6}\left(s^{2}\right)^{4}-\left(90\left(\Gamma_{1}\right)^{4}\right.$ $\left.+130\left(\Gamma_{2}\right)\left(\Gamma_{1}\right)^{3}+79\left(\Gamma_{2}\right)^{2}\left(\Gamma_{1}\right)^{2}+130\left(\Gamma_{2}\right)^{3}\left(\Gamma_{1}\right)+90\left(\Gamma_{2}\right)^{4}\right)\left(s^{3}\right)^{8}\left(s^{2}\right)^{2}$ $\left.+\left(30\left(\Gamma_{1}\right)^{4}-2\left(\Gamma_{2}\right)\left(\Gamma_{1}\right)^{3}-65\left(\Gamma_{2}\right)^{2}\left(\Gamma_{1}\right)^{2}+30\left(\Gamma_{2}\right)^{3}\left(\Gamma_{1}\right)+63\left(\Gamma_{2}\right)^{4}\right)\left(s^{3}\right)^{10}\right)\left(s^{1}\right)^{2}$ $+\left(\left(s^{2}\right)^{2}-\left(s^{3}\right)^{2}\right)^{2}\left(3\left(\Gamma_{1}\right)^{2}\left(7\left(\Gamma_{1}\right)^{2}+30\left(\Gamma_{2}\right)\left(\Gamma_{1}\right)+30\left(\Gamma_{2}\right)^{2}\right)\left(s^{2}\right)^{8}-2\left(\Gamma_{1}\right)\left(42\left(\Gamma_{1}\right)^{3}\right.\right.$ $\left.+132\left(\Gamma_{2}\right)\left(\Gamma_{1}\right)^{2}+133\left(\Gamma_{2}\right)^{2}\left(\Gamma_{1}\right)+34\left(\Gamma_{2}\right)^{3}\right)\left(s^{3}\right)^{2}\left(s^{2}\right)^{6}+\left(126\left(\Gamma_{1}\right)^{4}+252\left(\Gamma_{2}\right)\left(\Gamma_{1}\right)^{3}\right.$ $\left.+208\left(\Gamma_{2}\right)^{2}\left(\Gamma_{1}\right)^{2}+82\left(\Gamma_{2}\right)^{3}\left(\Gamma_{1}\right)+\left(\Gamma_{2}\right)^{4}\right)\left(s^{3}\right)^{4}\left(s^{2}\right)^{4}-2\left(42\left(\Gamma_{1}\right)^{4}+36\left(\Gamma_{2}\right)\left(\Gamma_{1}\right)^{3}\right.$ $\left.-11\left(\Gamma_{2}\right)^{2}\left(\Gamma_{1}\right)^{2}+4\left(\Gamma_{2}\right)^{3}\left(\Gamma_{1}\right)+9\left(\Gamma_{2}\right)^{4}\right)\left(s^{3}\right)^{6}\left(s^{2}\right)^{2}+3\left(\left(\Gamma_{1}\right)+\left(\Gamma_{2}\right)\right)^{2}\left(7\left(\Gamma_{1}\right)^{2}\right.$ $\left.\left.\left.-16\left(\Gamma_{2}\right)\left(\Gamma_{1}\right)+7\left(\Gamma_{2}\right)^{2}\right)\left(s^{3}\right)^{8}\right)\right)$,

$v\left(s^{1}, s^{2}, s^{3}\right)=\left(\Gamma_{2}\right)^{4}\left(18162\left(\Gamma_{1}\right)^{4}+36324\left(\Gamma_{2}\right)\left(\Gamma_{1}\right)^{3}+23662\left(\Gamma_{2}\right)^{2}\left(\Gamma_{1}\right)^{2}+5500\left(\Gamma_{2}\right)^{3}\left(\Gamma_{1}\right)\right.$ $\left.+441\left(\Gamma_{2}\right)^{4}\right)\left(s^{1}\right)^{24}-4\left(\Gamma_{2}\right)^{3}\left(\left(-6696\left(\Gamma_{1}\right)^{5}+18667\left(\Gamma_{2}\right)\left(\Gamma_{1}\right)^{4}+51328\left(\Gamma_{2}\right)^{2}\left(\Gamma_{1}\right)^{3}\right.\right.$ $\left.+33810\left(\Gamma_{2}\right)^{3}\left(\Gamma_{1}\right)^{2}+7152\left(\Gamma_{2}\right)^{4}\left(\Gamma_{1}\right)+630\left(\Gamma_{2}\right)^{5}\right)\left(s^{2}\right)^{2}+\left(6696\left(\Gamma_{1}\right)^{5}\right.$ $+52147\left(\Gamma_{2}\right)\left(\Gamma_{1}\right)^{4}+90300\left(\Gamma_{2}\right)^{2}\left(\Gamma_{1}\right)^{3}+58788\left(\Gamma_{2}\right)^{3}\left(\Gamma_{1}\right)^{2}+14632\left(\Gamma_{2}\right)^{4}\left(\Gamma_{1}\right)$ $\left.\left.+1323\left(\Gamma_{2}\right)^{5}\right)\left(s^{3}\right)^{2}\right)\left(s^{1}\right)^{22}+2\left(\Gamma_{2}\right)^{2}\left(\left(5440\left(\Gamma_{1}\right)^{6}-77152\left(\Gamma_{2}\right)\left(\Gamma_{1}\right)^{5}+41851\left(\Gamma_{2}\right)^{2}\left(\Gamma_{1}\right)^{4}\right.\right.$ $\left.+247924\left(\Gamma_{2}\right)^{3}\left(\Gamma_{1}\right)^{3}+163356\left(\Gamma_{2}\right)^{4}\left(\Gamma_{1}\right)^{2}+28340\left(\Gamma_{2}\right)^{5}\left(\Gamma_{1}\right)+3018\left(\Gamma_{2}\right)^{6}\right)\left(s^{2}\right)^{4}$ $-4\left(2720\left(\Gamma_{1}\right)^{6}+8160\left(\Gamma_{2}\right)\left(\Gamma_{1}\right)^{5}-81098\left(\Gamma_{2}\right)^{2}\left(\Gamma_{1}\right)^{4}-175796\left(\Gamma_{2}\right)^{3}\left(\Gamma_{1}\right)^{3}\right.$ $\left.-116084\left(\Gamma_{2}\right)^{4}\left(\Gamma_{1}\right)^{2}-26826\left(\Gamma_{2}\right)^{5}\left(\Gamma_{1}\right)-2835\left(\Gamma_{2}\right)^{6}\right)\left(s^{3}\right)^{2}\left(s^{2}\right)^{2}+\left(5440\left(\Gamma_{1}\right)^{6}\right.$ $+109792\left(\Gamma_{2}\right)\left(\Gamma_{1}\right)^{5}+509211\left(\Gamma_{2}\right)^{2}\left(\Gamma_{1}\right)^{4}+799800\left(\Gamma_{2}\right)^{3}\left(\Gamma_{1}\right)^{3}+523810\left(\Gamma_{2}\right)^{4}\left(\Gamma_{1}\right)^{2}$ $\left.\left.+140404\left(\Gamma_{2}\right)^{5}\left(\Gamma_{1}\right)+14553\left(\Gamma_{2}\right)^{6}\right)\left(s^{3}\right)^{4}\right)\left(s^{1}\right)^{20}-4\left(\Gamma_{2}\right)\left(\left(-254\left(\Gamma_{1}\right)^{7}+16194\left(\Gamma_{2}\right)\left(\Gamma_{1}\right)^{6}\right.\right.$ $-94894\left(\Gamma_{2}\right)^{2}\left(\Gamma_{1}\right)^{5}-18585\left(\Gamma_{2}\right)^{3}\left(\Gamma_{1}\right)^{4}+169694\left(\Gamma_{2}\right)^{4}\left(\Gamma_{1}\right)^{3}+110528\left(\Gamma_{2}\right)^{5}\left(\Gamma_{1}\right)^{2}$ $\left.+11286\left(\Gamma_{2}\right)^{6}\left(\Gamma_{1}\right)+1950\left(\Gamma_{2}\right)^{7}\right)\left(s^{2}\right)^{6}+2\left(381\left(\Gamma_{1}\right)^{7}-7208\left(\Gamma_{2}\right)\left(\Gamma_{1}\right)^{6}-52098\left(\Gamma_{2}\right)^{2}\left(\Gamma_{1}\right)^{5}\right.$ $+101802\left(\Gamma_{2}\right)^{3}\left(\Gamma_{1}\right)^{4}+298535\left(\Gamma_{2}\right)^{4}\left(\Gamma_{1}\right)^{3}+197888\left(\Gamma_{2}\right)^{5}\left(\Gamma_{1}\right)^{2}+40384\left(\Gamma_{2}\right)^{6}\left(\Gamma_{1}\right)$ $\left.+5745\left(\Gamma_{2}\right)^{7}\right)\left(s^{3}\right)^{2}\left(s^{2}\right)^{4}+2\left(-381\left(\Gamma_{1}\right)^{7}-9875\left(\Gamma_{2}\right)\left(\Gamma_{1}\right)^{6}+849\left(\Gamma_{2}\right)^{2}\left(\Gamma_{1}\right)^{5}\right.$ $+240837\left(\Gamma_{2}\right)^{3}\left(\Gamma_{1}\right)^{4}+472158\left(\Gamma_{2}\right)^{4}\left(\Gamma_{1}\right)^{3}+317954\left(\Gamma_{2}\right)^{5}\left(\Gamma_{1}\right)^{2}+81570\left(\Gamma_{2}\right)^{6}\left(\Gamma_{1}\right)$ $\left.+11025\left(\Gamma_{2}\right)^{7}\right)\left(s^{3}\right)^{4}\left(s^{2}\right)^{2}+\left(254\left(\Gamma_{1}\right)^{7}+17972\left(\Gamma_{2}\right)\left(\Gamma_{1}\right)^{6}+197392\left(\Gamma_{2}\right)^{2}\left(\Gamma_{1}\right)^{5}\right.$ $+707685\left(\Gamma_{2}\right)^{3}\left(\Gamma_{1}\right)^{4}+1037676\left(\Gamma_{2}\right)^{4}\left(\Gamma_{1}\right)^{3}+687120\left(\Gamma_{2}\right)^{5}\left(\Gamma_{1}\right)^{2}+199760\left(\Gamma_{2}\right)^{6}\left(\Gamma_{1}\right)$ $\left.\left.+24255\left(\Gamma_{2}\right)^{7}\right)\left(s^{3}\right)^{6}\right)\left(s^{1}\right)^{18}+\left(\left(\left(\Gamma_{1}\right)^{8}-6588\left(\Gamma_{2}\right)\left(\Gamma_{1}\right)^{7}+170110\left(\Gamma_{2}\right)^{2}\left(\Gamma_{1}\right)^{6}\right.\right.$ $-511988\left(\Gamma_{2}\right)^{3}\left(\Gamma_{1}\right)^{5}-292570\left(\Gamma_{2}\right)^{4}\left(\Gamma_{1}\right)^{4}+590480\left(\Gamma_{2}\right)^{5}\left(\Gamma_{1}\right)^{3}+397764\left(\Gamma_{2}\right)^{6}\left(\Gamma_{1}\right)^{2}$ $\left.-9088\left(\Gamma_{2}\right)^{7}\left(\Gamma_{1}\right)+5806\left(\Gamma_{2}\right)^{8}\right)\left(s^{2}\right)^{8}-4\left(\left(\Gamma_{1}\right)^{8}-3292\left(\Gamma_{2}\right)\left(\Gamma_{1}\right)^{7}+29964\left(\Gamma_{2}\right)^{2}\left(\Gamma_{1}\right)^{6}\right.$
$+192466\left(\Gamma_{2}\right)^{3}\left(\Gamma_{1}\right)^{5}-112305\left(\Gamma_{2}\right)^{4}\left(\Gamma_{1}\right)^{4}-558040\left(\Gamma_{2}\right)^{5}\left(\Gamma_{1}\right)^{3}-379280\left(\Gamma_{2}\right)^{6}\left(\Gamma_{1}\right)^{2}$ $\left.-59154\left(\Gamma_{2}\right)^{7}\left(\Gamma_{1}\right)-13650\left(\Gamma_{2}\right)^{8}\right)\left(s^{3}\right)^{2}\left(s^{2}\right)^{6}+2\left(3\left(\Gamma_{1}\right)^{8}+12\left(\Gamma_{2}\right)\left(\Gamma_{1}\right)^{7}-27182\left(\Gamma_{2}\right)^{2}\left(\Gamma_{1}\right)^{6}\right.$ $-81588\left(\Gamma_{2}\right)^{3}\left(\Gamma_{1}\right)^{5}+1041151\left(\Gamma_{2}\right)^{4}\left(\Gamma_{1}\right)^{4}+2218296\left(\Gamma_{2}\right)^{5}\left(\Gamma_{1}\right)^{3}+1511656\left(\Gamma_{2}\right)^{6}\left(\Gamma_{1}\right)^{2}$ $\left.+388908\left(\Gamma_{2}\right)^{7}\left(\Gamma_{1}\right)+78210\left(\Gamma_{2}\right)^{8}\right)\left(s^{3}\right)^{4}\left(s^{2}\right)^{4}-4\left(\left(\Gamma_{1}\right)^{8}+3300\left(\Gamma_{2}\right)\left(\Gamma_{1}\right)^{7}\right.$ $+53036\left(\Gamma_{2}\right)^{2}\left(\Gamma_{1}\right)^{6}+56506\left(\Gamma_{2}\right)^{3}\left(\Gamma_{1}\right)^{5}-509885\left(\Gamma_{2}\right)^{4}\left(\Gamma_{1}\right)^{4}-1101284\left(\Gamma_{2}\right)^{5}\left(\Gamma_{1}\right)^{3}$ $\left.-785030\left(\Gamma_{2}\right)^{6}\left(\Gamma_{1}\right)^{2}-234000\left(\Gamma_{2}\right)^{7}\left(\Gamma_{1}\right)-47250\left(\Gamma_{2}\right)^{8}\right)\left(s^{3}\right)^{6}\left(s^{2}\right)^{2}+\left(\left(\Gamma_{1}\right)^{8}\right.$ $+6596\left(\Gamma_{2}\right)\left(\Gamma_{1}\right)^{7}+216254\left(\Gamma_{2}\right)^{2}\left(\Gamma_{1}\right)^{6}+1671052\left(\Gamma_{2}\right)^{3}\left(\Gamma_{1}\right)^{5}+5049670\left(\Gamma_{2}\right)^{4}\left(\Gamma_{1}\right)^{4}$ $\left.\left.+6991956\left(\Gamma_{2}\right)^{5}\left(\Gamma_{1}\right)^{3}+4680810\left(\Gamma_{2}\right)^{6}\left(\Gamma_{1}\right)^{2}+1489620\left(\Gamma_{2}\right)^{7}\left(\Gamma_{1}\right)+218295\left(\Gamma_{2}\right)^{8}\right)\left(s^{3}\right)^{8}\right)\left(s^{1}\right)^{16}$ $-8\left(\left(5\left(\Gamma_{1}\right)^{8}-2711\left(\Gamma_{2}\right)\left(\Gamma_{1}\right)^{7}+33088\left(\Gamma_{2}\right)^{2}\left(\Gamma_{1}\right)^{6}-47351\left(\Gamma_{2}\right)^{3}\left(\Gamma_{1}\right)^{5}-46857\left(\Gamma_{2}\right)^{4}\left(\Gamma_{1}\right)^{4}\right.\right.$ $\left.+38643\left(\Gamma_{2}\right)^{5}\left(\Gamma_{1}\right)^{3}+39638\left(\Gamma_{2}\right)^{6}\left(\Gamma_{1}\right)^{2}-6061\left(\Gamma_{2}\right)^{7}\left(\Gamma_{1}\right)+305\left(\Gamma_{2}\right)^{8}\right)\left(s^{2}\right)^{10}+\left(-15\left(\Gamma_{1}\right)^{8}\right.$ $+6665\left(\Gamma_{2}\right)\left(\Gamma_{1}\right)^{7}-26848\left(\Gamma_{2}\right)^{2}\left(\Gamma_{1}\right)^{6}-95484\left(\Gamma_{2}\right)^{3}\left(\Gamma_{1}\right)^{5}-16877\left(\Gamma_{2}\right)^{4}\left(\Gamma_{1}\right)^{4}$ $+123073\left(\Gamma_{2}\right)^{5}\left(\Gamma_{1}\right)^{3}+103952\left(\Gamma_{2}\right)^{6}\left(\Gamma_{1}\right)^{2}+2214\left(\Gamma_{2}\right)^{7}\left(\Gamma_{1}\right)$ $\left.+4606\left(\Gamma_{2}\right)^{8}\right)\left(s^{3}\right)^{2}\left(s^{2}\right)^{8}+\left(10\left(\Gamma_{1}\right)^{8}-6480\left(\Gamma_{2}\right)\left(\Gamma_{1}\right)^{7}-15081\left(\Gamma_{2}\right)^{2}\left(\Gamma_{1}\right)^{6}\right.$ $-43768\left(\Gamma_{2}\right)^{3}\left(\Gamma_{1}\right)^{5}+134234\left(\Gamma_{2}\right)^{4}\left(\Gamma_{1}\right)^{4}+334538\left(\Gamma_{2}\right)^{5}\left(\Gamma_{1}\right)^{3}+251045\left(\Gamma_{2}\right)^{6}\left(\Gamma_{1}\right)^{2}$ $\left.+65278\left(\Gamma_{2}\right)^{7}\left(\Gamma_{1}\right)+19500\left(\Gamma_{2}\right)^{8}\right)\left(s^{3}\right)^{4}\left(s^{2}\right)^{6}+\left(10\left(\Gamma_{1}\right)^{8}+6560\left(\Gamma_{2}\right)\left(\Gamma_{1}\right)^{7}+30559\left(\Gamma_{2}\right)^{2}\left(\Gamma_{1}\right)^{6}\right.$ $+89922\left(\Gamma_{2}\right)^{3}\left(\Gamma_{1}\right)^{5}+354359\left(\Gamma_{2}\right)^{4}\left(\Gamma_{1}\right)^{4}+565818\left(\Gamma_{2}\right)^{5}\left(\Gamma_{1}\right)^{3}+400660\left(\Gamma_{2}\right)^{6}\left(\Gamma_{1}\right)^{2}$ $\left.+143928\left(\Gamma_{2}\right)^{7}\left(\Gamma_{1}\right)+40140\left(\Gamma_{2}\right)^{8}\right)\left(s^{3}\right)^{6}\left(s^{2}\right)^{4}-\left(15\left(\Gamma_{1}\right)^{8}+6785\left(\Gamma_{2}\right)\left(\Gamma_{1}\right)^{7}\right.$ $+73923\left(\Gamma_{2}\right)^{2}\left(\Gamma_{1}\right)^{6}+206409\left(\Gamma_{2}\right)^{3}\left(\Gamma_{1}\right)^{5}+176502\left(\Gamma_{2}\right)^{4}\left(\Gamma_{1}\right)^{4}+6816\left(\Gamma_{2}\right)^{5}\left(\Gamma_{1}\right)^{3}$ $\left.-45206\left(\Gamma_{2}\right)^{6}\left(\Gamma_{1}\right)^{2}-38520\left(\Gamma_{2}\right)^{7}\left(\Gamma_{1}\right)-28350\left(\Gamma_{2}\right)^{8}\right)\left(s^{3}\right)^{8}\left(s^{2}\right)^{2}+\left(5\left(\Gamma_{1}\right)^{8}+2751\left(\Gamma_{2}\right)\left(\Gamma_{1}\right)^{7}\right.$ $+52205\left(\Gamma_{2}\right)^{2}\left(\Gamma_{1}\right)^{6}+303090\left(\Gamma_{2}\right)^{3}\left(\Gamma_{1}\right)^{5}+781453\left(\Gamma_{2}\right)^{4}\left(\Gamma_{1}\right)^{4}+1004364\left(\Gamma_{2}\right)^{5}\left(\Gamma_{1}\right)^{3}$ $\left.\left.+669468\left(\Gamma_{2}\right)^{6}\left(\Gamma_{1}\right)^{2}+236280\left(\Gamma_{2}\right)^{7}\left(\Gamma_{1}\right)+43659\left(\Gamma_{2}\right)^{8}\right)\left(s^{3}\right)^{10}\right)\left(s^{1}\right)^{14}+4\left(\left(129\left(\Gamma_{1}\right)^{8}\right.\right.$ $-10986\left(\Gamma_{2}\right)\left(\Gamma_{1}\right)^{7}+73702\left(\Gamma_{2}\right)^{2}\left(\Gamma_{1}\right)^{6}-12074\left(\Gamma_{2}\right)^{3}\left(\Gamma_{1}\right)^{5}-91911\left(\Gamma_{2}\right)^{4}\left(\Gamma_{1}\right)^{4}$ $\left.-12074\left(\Gamma_{2}\right)^{5}\left(\Gamma_{1}\right)^{3}+73702\left(\Gamma_{2}\right)^{6}\left(\Gamma_{1}\right)^{2}-10986\left(\Gamma_{2}\right)^{7}\left(\Gamma_{1}\right)+129\left(\Gamma_{2}\right)^{8}\right)\left(s^{2}\right)^{12}-2\left(187\left(\Gamma_{1}\right)^{8}\right.$ $-16428\left(\Gamma_{2}\right)\left(\Gamma_{1}\right)^{7}+24952\left(\Gamma_{2}\right)^{2}\left(\Gamma_{1}\right)^{6}+32977\left(\Gamma_{2}\right)^{3}\left(\Gamma_{1}\right)^{5}+26252\left(\Gamma_{2}\right)^{4}\left(\Gamma_{1}\right)^{4}$ $\left.-5078\left(\Gamma_{2}\right)^{5}\left(\Gamma_{1}\right)^{3}-34108\left(\Gamma_{2}\right)^{6}\left(\Gamma_{1}\right)^{2}+17517\left(\Gamma_{2}\right)^{7}\left(\Gamma_{1}\right)-1525\left(\Gamma_{2}\right)^{8}\right)\left(s^{3}\right)^{2}\left(s^{2}\right)^{10}$ $+\left(335\left(\Gamma_{1}\right)^{8}-32858\left(\Gamma_{2}\right)\left(\Gamma_{1}\right)^{7}-46092\left(\Gamma_{2}\right)^{2}\left(\Gamma_{1}\right)^{6}-110272\left(\Gamma_{2}\right)^{3}\left(\Gamma_{1}\right)^{5}+9459\left(\Gamma_{2}\right)^{4}\left(\Gamma_{1}\right)^{4}\right.$ $\left.+186738\left(\Gamma_{2}\right)^{5}\left(\Gamma_{1}\right)^{3}+225022\left(\Gamma_{2}\right)^{6}\left(\Gamma_{1}\right)^{2}+56068\left(\Gamma_{2}\right)^{7}\left(\Gamma_{1}\right)+26242\left(\Gamma_{2}\right)^{8}\right)\left(s^{3}\right)^{4}\left(s^{2}\right)^{8}$ $-2\left(90\left(\Gamma_{1}\right)^{8}+360\left(\Gamma_{2}\right)\left(\Gamma_{1}\right)^{7}+17424\left(\Gamma_{2}\right)^{2}\left(\Gamma_{1}\right)^{6}+51012\left(\Gamma_{2}\right)^{3}\left(\Gamma_{1}\right)^{5}-20391\left(\Gamma_{2}\right)^{4}\left(\Gamma_{1}\right)^{4}\right.$ $\left.-125382\left(\Gamma_{2}\right)^{5}\left(\Gamma_{1}\right)^{3}-137135\left(\Gamma_{2}\right)^{6}\left(\Gamma_{1}\right)^{2}-66002\left(\Gamma_{2}\right)^{7}\left(\Gamma_{1}\right)-27300\left(\Gamma_{2}\right)^{8}\right)\left(s^{3}\right)^{6}\left(s^{2}\right)^{6}$ $+\left(335\left(\Gamma_{1}\right)^{8}+35538\left(\Gamma_{2}\right)\left(\Gamma_{1}\right)^{7}+193294\left(\Gamma_{2}\right)^{2}\left(\Gamma_{1}\right)^{6}+542498\left(\Gamma_{2}\right)^{3}\left(\Gamma_{1}\right)^{5}+1042919\left(\Gamma_{2}\right)^{4}\left(\Gamma_{1}\right)^{4}\right.$ $\left.+1200768\left(\Gamma_{2}\right)^{5}\left(\Gamma_{1}\right)^{3}+832300\left(\Gamma_{2}\right)^{6}\left(\Gamma_{1}\right)^{2}+379092\left(\Gamma_{2}\right)^{7}\left(\Gamma_{1}\right)+115290\left(\Gamma_{2}\right)^{8}\right)\left(s^{3}\right)^{8}\left(s^{2}\right)^{4}$ $-2\left(187\left(\Gamma_{1}\right)^{8}+17924\left(\Gamma_{2}\right)\left(\Gamma_{1}\right)^{7}+145184\left(\Gamma_{2}\right)^{2}\left(\Gamma_{1}\right)^{6}+472195\left(\Gamma_{2}\right)^{3}\left(\Gamma_{1}\right)^{5}+823717\left(\Gamma_{2}\right)^{4}\left(\Gamma_{1}\right)^{4}\right.$ $\left.+864808\left(\Gamma_{2}\right)^{5}\left(\Gamma_{1}\right)^{3}+533372\left(\Gamma_{2}\right)^{6}\left(\Gamma_{1}\right)^{2}+135828\left(\Gamma_{2}\right)^{7}\left(\Gamma_{1}\right)-13230\left(\Gamma_{2}\right)^{8}\right)\left(s^{3}\right)^{10}\left(s^{2}\right)^{2}$ $+\left(129\left(\Gamma_{1}\right)^{8}+12018\left(\Gamma_{2}\right)\left(\Gamma_{1}\right)^{7}+154216\left(\Gamma_{2}\right)^{2}\left(\Gamma_{1}\right)^{6}+692216\left(\Gamma_{2}\right)^{3}\left(\Gamma_{1}\right)^{5}+1467529\left(\Gamma_{2}\right)^{4}\left(\Gamma_{1}\right)^{4}\right.$ $\left.\left.+1630944\left(\Gamma_{2}\right)^{5}\left(\Gamma_{1}\right)^{3}+1019046\left(\Gamma_{2}\right)^{6}\left(\Gamma_{1}\right)^{2}+407484\left(\Gamma_{2}\right)^{7}\left(\Gamma_{1}\right)+101871\left(\Gamma_{2}\right)^{8}\right)\left(s^{3}\right)^{12}\right)\left(s^{1}\right)^{12}$ $-8\left(\left(305\left(\Gamma_{1}\right)^{8}-6061\left(\Gamma_{2}\right)\left(\Gamma_{1}\right)^{7}+39638\left(\Gamma_{2}\right)^{2}\left(\Gamma_{1}\right)^{6}+38643\left(\Gamma_{2}\right)^{3}\left(\Gamma_{1}\right)^{5}-46857\left(\Gamma_{2}\right)^{4}\left(\Gamma_{1}\right)^{4}\right.\right.$ $\left.-47351\left(\Gamma_{2}\right)^{5}\left(\Gamma_{1}\right)^{3}+33088\left(\Gamma_{2}\right)^{6}\left(\Gamma_{1}\right)^{2}-2711\left(\Gamma_{2}\right)^{7}\left(\Gamma_{1}\right)+5\left(\Gamma_{2}\right)^{8}\right)\left(s^{2}\right)^{14}+\left(-1525\left(\Gamma_{1}\right)^{8}\right.$ $+17517\left(\Gamma_{2}\right)\left(\Gamma_{1}\right)^{7}-34108\left(\Gamma_{2}\right)^{2}\left(\Gamma_{1}\right)^{6}-5078\left(\Gamma_{2}\right)^{3}\left(\Gamma_{1}\right)^{5}+26252\left(\Gamma_{2}\right)^{4}\left(\Gamma_{1}\right)^{4}+32977\left(\Gamma_{2}\right)^{5}\left(\Gamma_{1}\right)^{3}$ $\left.+24952\left(\Gamma_{2}\right)^{6}\left(\Gamma_{1}\right)^{2}-16428\left(\Gamma_{2}\right)^{7}\left(\Gamma_{1}\right)+187\left(\Gamma_{2}\right)^{8}\right)\left(s^{3}\right)^{2}\left(s^{2}\right)^{12}+\left(2745\left(\Gamma_{1}\right)^{8}-21651\left(\Gamma_{2}\right)\left(\Gamma_{1}\right)^{7}\right.$ $-40747\left(\Gamma_{2}\right)^{2}\left(\Gamma_{1}\right)^{6}-143169\left(\Gamma_{2}\right)^{3}\left(\Gamma_{1}\right)^{5}-189502\left(\Gamma_{2}\right)^{4}\left(\Gamma_{1}\right)^{4}-143169\left(\Gamma_{2}\right)^{5}\left(\Gamma_{1}\right)^{3}$ $\left.-40747\left(\Gamma_{2}\right)^{6}\left(\Gamma_{1}\right)^{2}-21651\left(\Gamma_{2}\right)^{7}\left(\Gamma_{1}\right)+2745\left(\Gamma_{2}\right)^{8}\right)\left(s^{3}\right)^{4}\left(s^{2}\right)^{10}+\left(-1525\left(\Gamma_{1}\right)^{8}\right.$ 
$+24675\left(\Gamma_{2}\right)\left(\Gamma_{1}\right)^{7}+85897\left(\Gamma_{2}\right)^{2}\left(\Gamma_{1}\right)^{6}+172086\left(\Gamma_{2}\right)^{3}\left(\Gamma_{1}\right)^{5}+235132\left(\Gamma_{2}\right)^{4}\left(\Gamma_{1}\right)^{4}$ $\left.+232359\left(\Gamma_{2}\right)^{5}\left(\Gamma_{1}\right)^{3}+174766\left(\Gamma_{2}\right)^{6}\left(\Gamma_{1}\right)^{2}+74142\left(\Gamma_{2}\right)^{7}\left(\Gamma_{1}\right)+22642\left(\Gamma_{2}\right)^{8}\right)\left(s^{3}\right)^{6}\left(s^{2}\right)^{8}$ $-\left(1525\left(\Gamma_{1}\right)^{8}+36875\left(\Gamma_{2}\right)\left(\Gamma_{1}\right)^{7}+129528\left(\Gamma_{2}\right)^{2}\left(\Gamma_{1}\right)^{6}+260279\left(\Gamma_{2}\right)^{3}\left(\Gamma_{1}\right)^{5}+307218\left(\Gamma_{2}\right)^{4}\left(\Gamma_{1}\right)^{4}\right.$ $\left.+243776\left(\Gamma_{2}\right)^{5}\left(\Gamma_{1}\right)^{3}+104799\left(\Gamma_{2}\right)^{6}\left(\Gamma_{1}\right)^{2}+11132\left(\Gamma_{2}\right)^{7}\left(\Gamma_{1}\right)-13650\left(\Gamma_{2}\right)^{8}\right)\left(s^{3}\right)^{8}\left(s^{2}\right)^{6}$ $+\left(2745\left(\Gamma_{1}\right)^{8}+43611\left(\Gamma_{2}\right)\left(\Gamma_{1}\right)^{7}+187670\left(\Gamma_{2}\right)^{2}\left(\Gamma_{1}\right)^{6}+507078\left(\Gamma_{2}\right)^{3}\left(\Gamma_{1}\right)^{5}+865073\left(\Gamma_{2}\right)^{4}\left(\Gamma_{1}\right)^{4}\right.$ $\left.+913416\left(\Gamma_{2}\right)^{5}\left(\Gamma_{1}\right)^{3}+603764\left(\Gamma_{2}\right)^{6}\left(\Gamma_{1}\right)^{2}+256536\left(\Gamma_{2}\right)^{7}\left(\Gamma_{1}\right)+64134\left(\Gamma_{2}\right)^{8}\right)\left(s^{3}\right)^{10}\left(s^{2}\right)^{4}$ $-\left(1525\left(\Gamma_{1}\right)^{8}+29717\left(\Gamma_{2}\right)\left(\Gamma_{1}\right)^{7}+199427\left(\Gamma_{2}\right)^{2}\left(\Gamma_{1}\right)^{6}+652827\left(\Gamma_{2}\right)^{3}\left(\Gamma_{1}\right)^{5}\right.$ $+1179823\left(\Gamma_{2}\right)^{4}\left(\Gamma_{1}\right)^{4}+1257844\left(\Gamma_{2}\right)^{5}\left(\Gamma_{1}\right)^{3}+787864\left(\Gamma_{2}\right)^{6}\left(\Gamma_{1}\right)^{2}+241668\left(\Gamma_{2}\right)^{7}\left(\Gamma_{1}\right)$ $\left.+13230\left(\Gamma_{2}\right)^{8}\right)\left(s^{3}\right)^{12}\left(s^{2}\right)^{2}+\left(305\left(\Gamma_{1}\right)^{8}+8501\left(\Gamma_{2}\right)\left(\Gamma_{1}\right)^{7}+90605\left(\Gamma_{2}\right)^{2}\left(\Gamma_{1}\right)^{6}+343546\left(\Gamma_{2}\right)^{3}\left(\Gamma_{1}\right)^{5}\right.$ $+587983\left(\Gamma_{2}\right)^{4}\left(\Gamma_{1}\right)^{4}+495468\left(\Gamma_{2}\right)^{5}\left(\Gamma_{1}\right)^{3}+237960\left(\Gamma_{2}\right)^{6}\left(\Gamma_{1}\right)^{2}+112992\left(\Gamma_{2}\right)^{7}\left(\Gamma_{1}\right)$ $\left.\left.+43659\left(\Gamma_{2}\right)^{8}\right)\left(s^{3}\right)^{14}\right)\left(s^{1}\right)^{10}+\left(\left(5806\left(\Gamma_{1}\right)^{8}-9088\left(\Gamma_{2}\right)\left(\Gamma_{1}\right)^{7}+397764\left(\Gamma_{2}\right)^{2}\left(\Gamma_{1}\right)^{6}\right.\right.$ $+590480\left(\Gamma_{2}\right)^{3}\left(\Gamma_{1}\right)^{5}-292570\left(\Gamma_{2}\right)^{4}\left(\Gamma_{1}\right)^{4}-511988\left(\Gamma_{2}\right)^{5}\left(\Gamma_{1}\right)^{3}+170110\left(\Gamma_{2}\right)^{6}\left(\Gamma_{1}\right)^{2}$ $\left.-6588\left(\Gamma_{2}\right)^{7}\left(\Gamma_{1}\right)+\left(\Gamma_{2}\right)^{8}\right)\left(s^{2}\right)^{16}-8\left(4606\left(\Gamma_{1}\right)^{8}+2214\left(\Gamma_{2}\right)\left(\Gamma_{1}\right)^{7}+103952\left(\Gamma_{2}\right)^{2}\left(\Gamma_{1}\right)^{6}\right.$ $+123073\left(\Gamma_{2}\right)^{3}\left(\Gamma_{1}\right)^{5}-16877\left(\Gamma_{2}\right)^{4}\left(\Gamma_{1}\right)^{4}-95484\left(\Gamma_{2}\right)^{5}\left(\Gamma_{1}\right)^{3}-26848\left(\Gamma_{2}\right)^{6}\left(\Gamma_{1}\right)^{2}$ $\left.+6665\left(\Gamma_{2}\right)^{7}\left(\Gamma_{1}\right)-15\left(\Gamma_{2}\right)^{8}\right)\left(s^{3}\right)^{2}\left(s^{2}\right)^{14}+4\left(26242\left(\Gamma_{1}\right)^{8}+56068\left(\Gamma_{2}\right)\left(\Gamma_{1}\right)^{7}+225022\left(\Gamma_{2}\right)^{2}\left(\Gamma_{1}\right)^{6}\right.$ $+186738\left(\Gamma_{2}\right)^{3}\left(\Gamma_{1}\right)^{5}+9459\left(\Gamma_{2}\right)^{4}\left(\Gamma_{1}\right)^{4}-110272\left(\Gamma_{2}\right)^{5}\left(\Gamma_{1}\right)^{3}-46092\left(\Gamma_{2}\right)^{6}\left(\Gamma_{1}\right)^{2}$ $\left.-32858\left(\Gamma_{2}\right)^{7}\left(\Gamma_{1}\right)+335\left(\Gamma_{2}\right)^{8}\right)\left(s^{3}\right)^{4}\left(s^{2}\right)^{12}-8\left(22642\left(\Gamma_{1}\right)^{8}+74142\left(\Gamma_{2}\right)\left(\Gamma_{1}\right)^{7}\right.$ $+174766\left(\Gamma_{2}\right)^{2}\left(\Gamma_{1}\right)^{6}+232359\left(\Gamma_{2}\right)^{3}\left(\Gamma_{1}\right)^{5}+235132\left(\Gamma_{2}\right)^{4}\left(\Gamma_{1}\right)^{4}+172086\left(\Gamma_{2}\right)^{5}\left(\Gamma_{1}\right)^{3}$ $\left.+85897\left(\Gamma_{2}\right)^{6}\left(\Gamma_{1}\right)^{2}+24675\left(\Gamma_{2}\right)^{7}\left(\Gamma_{1}\right)-1525\left(\Gamma_{2}\right)^{8}\right)\left(s^{3}\right)^{6}\left(s^{2}\right)^{10}+4\left(53605\left(\Gamma_{1}\right)^{8}\right.$ $+214420\left(\Gamma_{2}\right)\left(\Gamma_{1}\right)^{7}+524004\left(\Gamma_{2}\right)^{2}\left(\Gamma_{1}\right)^{6}+821542\left(\Gamma_{2}\right)^{3}\left(\Gamma_{1}\right)^{5}+970311\left(\Gamma_{2}\right)^{4}\left(\Gamma_{1}\right)^{4}$ $\left.+821542\left(\Gamma_{2}\right)^{5}\left(\Gamma_{1}\right)^{3}+524004\left(\Gamma_{2}\right)^{6}\left(\Gamma_{1}\right)^{2}+214420\left(\Gamma_{2}\right)^{7}\left(\Gamma_{1}\right)+53605\left(\Gamma_{2}\right)^{8}\right)\left(s^{3}\right)^{8}\left(s^{2}\right)^{8}$ $-8\left(22642\left(\Gamma_{1}\right)^{8}+106994\left(\Gamma_{2}\right)\left(\Gamma_{1}\right)^{7}+289748\left(\Gamma_{2}\right)^{2}\left(\Gamma_{1}\right)^{6}+527207\left(\Gamma_{2}\right)^{3}\left(\Gamma_{1}\right)^{5}\right.$ $+684797\left(\Gamma_{2}\right)^{4}\left(\Gamma_{1}\right)^{4}+613154\left(\Gamma_{2}\right)^{5}\left(\Gamma_{1}\right)^{3}+355325\left(\Gamma_{2}\right)^{6}\left(\Gamma_{1}\right)^{2}+120332\left(\Gamma_{2}\right)^{7}\left(\Gamma_{1}\right)$ $\left.+13650\left(\Gamma_{2}\right)^{8}\right)\left(s^{3}\right)^{10}\left(s^{2}\right)^{6}+4\left(26242\left(\Gamma_{1}\right)^{8}+153868\left(\Gamma_{2}\right)\left(\Gamma_{1}\right)^{7}+567322\left(\Gamma_{2}\right)^{2}\left(\Gamma_{1}\right)^{6}\right.$ $+1455518\left(\Gamma_{2}\right)^{3}\left(\Gamma_{1}\right)^{5}+2325659\left(\Gamma_{2}\right)^{4}\left(\Gamma_{1}\right)^{4}+2288340\left(\Gamma_{2}\right)^{5}\left(\Gamma_{1}\right)^{3}+1406776\left(\Gamma_{2}\right)^{6}\left(\Gamma_{1}\right)^{2}$ $\left.+543228\left(\Gamma_{2}\right)^{7}\left(\Gamma_{1}\right)+115290\left(\Gamma_{2}\right)^{8}\right)\left(s^{3}\right)^{12}\left(s^{2}\right)^{4}-8\left(4606\left(\Gamma_{1}\right)^{8}+34634\left(\Gamma_{2}\right)\left(\Gamma_{1}\right)^{7}\right.$ $+217422\left(\Gamma_{2}\right)^{2}\left(\Gamma_{1}\right)^{6}+712081\left(\Gamma_{2}\right)^{3}\left(\Gamma_{1}\right)^{5}+1171968\left(\Gamma_{2}\right)^{4}\left(\Gamma_{1}\right)^{4}+1056732\left(\Gamma_{2}\right)^{5}\left(\Gamma_{1}\right)^{3}$ $\left.+569366\left(\Gamma_{2}\right)^{6}\left(\Gamma_{1}\right)^{2}+188280\left(\Gamma_{2}\right)^{7}\left(\Gamma_{1}\right)+28350\left(\Gamma_{2}\right)^{8}\right)\left(s^{3}\right)^{14}\left(s^{2}\right)^{2}+\left(5806\left(\Gamma_{1}\right)^{8}\right.$ $+55536\left(\Gamma_{2}\right)\left(\Gamma_{1}\right)^{7}+623948\left(\Gamma_{2}\right)^{2}\left(\Gamma_{1}\right)^{6}+2312088\left(\Gamma_{2}\right)^{3}\left(\Gamma_{1}\right)^{5}+3445990\left(\Gamma_{2}\right)^{4}\left(\Gamma_{1}\right)^{4}$ $\left.\left.+2035404\left(\Gamma_{2}\right)^{5}\left(\Gamma_{1}\right)^{3}+365730\left(\Gamma_{2}\right)^{6}\left(\Gamma_{1}\right)^{2}+256740\left(\Gamma_{2}\right)^{7}\left(\Gamma_{1}\right)+218295\left(\Gamma_{2}\right)^{8}\right)\left(s^{3}\right)^{16}\right)\left(s^{1}\right)^{8}$ $-4\left(\left(\Gamma_{1}\right)\left(1950\left(\Gamma_{1}\right)^{7}+11286\left(\Gamma_{2}\right)\left(\Gamma_{1}\right)^{6}+110528\left(\Gamma_{2}\right)^{2}\left(\Gamma_{1}\right)^{5}+169694\left(\Gamma_{2}\right)^{3}\left(\Gamma_{1}\right)^{4}\right.\right.$ $\left.-18585\left(\Gamma_{2}\right)^{4}\left(\Gamma_{1}\right)^{3}-94894\left(\Gamma_{2}\right)^{5}\left(\Gamma_{1}\right)^{2}+16194\left(\Gamma_{2}\right)^{6}\left(\Gamma_{1}\right)-254\left(\Gamma_{2}\right)^{7}\right)\left(s^{2}\right)^{18}-\left(13650\left(\Gamma_{1}\right)^{8}\right.$ $+59154\left(\Gamma_{2}\right)\left(\Gamma_{1}\right)^{7}+379280\left(\Gamma_{2}\right)^{2}\left(\Gamma_{1}\right)^{6}+558040\left(\Gamma_{2}\right)^{3}\left(\Gamma_{1}\right)^{5}+112305\left(\Gamma_{2}\right)^{4}\left(\Gamma_{1}\right)^{4}$ $\left.-192466\left(\Gamma_{2}\right)^{5}\left(\Gamma_{1}\right)^{3}-29964\left(\Gamma_{2}\right)^{6}\left(\Gamma_{1}\right)^{2}+3292\left(\Gamma_{2}\right)^{7}\left(\Gamma_{1}\right)-\left(\Gamma_{2}\right)^{8}\right)\left(s^{3}\right)^{2}\left(s^{2}\right)^{16}+2\left(19500\left(\Gamma_{1}\right)^{8}\right.$ $+65278\left(\Gamma_{2}\right)\left(\Gamma_{1}\right)^{7}+251045\left(\Gamma_{2}\right)^{2}\left(\Gamma_{1}\right)^{6}+334538\left(\Gamma_{2}\right)^{3}\left(\Gamma_{1}\right)^{5}+134234\left(\Gamma_{2}\right)^{4}\left(\Gamma_{1}\right)^{4}$ $\left.-43768\left(\Gamma_{2}\right)^{5}\left(\Gamma_{1}\right)^{3}-15081\left(\Gamma_{2}\right)^{6}\left(\Gamma_{1}\right)^{2}-6480\left(\Gamma_{2}\right)^{7}\left(\Gamma_{1}\right)+10\left(\Gamma_{2}\right)^{8}\right)\left(s^{3}\right)^{4}\left(s^{2}\right)^{14}-2\left(27300\left(\Gamma_{1}\right)^{8}\right.$ $+66002\left(\Gamma_{2}\right)\left(\Gamma_{1}\right)^{7}+137135\left(\Gamma_{2}\right)^{2}\left(\Gamma_{1}\right)^{6}+125382\left(\Gamma_{2}\right)^{3}\left(\Gamma_{1}\right)^{5}+20391\left(\Gamma_{2}\right)^{4}\left(\Gamma_{1}\right)^{4}$ $\left.-51012\left(\Gamma_{2}\right)^{5}\left(\Gamma_{1}\right)^{3}-17424\left(\Gamma_{2}\right)^{6}\left(\Gamma_{1}\right)^{2}-360\left(\Gamma_{2}\right)^{7}\left(\Gamma_{1}\right)-90\left(\Gamma_{2}\right)^{8}\right)\left(s^{3}\right)^{6}\left(s^{2}\right)^{12}+2\left(13650\left(\Gamma_{1}\right)^{8}\right.$ $-11132\left(\Gamma_{2}\right)\left(\Gamma_{1}\right)^{7}-104799\left(\Gamma_{2}\right)^{2}\left(\Gamma_{1}\right)^{6}-243776\left(\Gamma_{2}\right)^{3}\left(\Gamma_{1}\right)^{5}-307218\left(\Gamma_{2}\right)^{4}\left(\Gamma_{1}\right)^{4}$ $\left.-260279\left(\Gamma_{2}\right)^{5}\left(\Gamma_{1}\right)^{3}-129528\left(\Gamma_{2}\right)^{6}\left(\Gamma_{1}\right)^{2}-36875\left(\Gamma_{2}\right)^{7}\left(\Gamma_{1}\right)-1525\left(\Gamma_{2}\right)^{8}\right)\left(s^{3}\right)^{8}\left(s^{2}\right)^{10}$
$+2\left(13650\left(\Gamma_{1}\right)^{8}+120332\left(\Gamma_{2}\right)\left(\Gamma_{1}\right)^{7}+355325\left(\Gamma_{2}\right)^{2}\left(\Gamma_{1}\right)^{6}+613154\left(\Gamma_{2}\right)^{3}\left(\Gamma_{1}\right)^{5}\right.$ $+684797\left(\Gamma_{2}\right)^{4}\left(\Gamma_{1}\right)^{4}+527207\left(\Gamma_{2}\right)^{5}\left(\Gamma_{1}\right)^{3}+289748\left(\Gamma_{2}\right)^{6}\left(\Gamma_{1}\right)^{2}+106994\left(\Gamma_{2}\right)^{7}\left(\Gamma_{1}\right)$ $\left.+22642\left(\Gamma_{2}\right)^{8}\right)\left(s^{3}\right)^{10}\left(s^{2}\right)^{8}-2\left(27300\left(\Gamma_{1}\right)^{8}+152398\left(\Gamma_{2}\right)\left(\Gamma_{1}\right)^{7}+439521\left(\Gamma_{2}\right)^{2}\left(\Gamma_{1}\right)^{6}\right.$ $+840186\left(\Gamma_{2}\right)^{3}\left(\Gamma_{1}\right)^{5}+1051436\left(\Gamma_{2}\right)^{4}\left(\Gamma_{1}\right)^{4}+840186\left(\Gamma_{2}\right)^{5}\left(\Gamma_{1}\right)^{3}+439521\left(\Gamma_{2}\right)^{6}\left(\Gamma_{1}\right)^{2}$ $\left.+152398\left(\Gamma_{2}\right)^{7}\left(\Gamma_{1}\right)+27300\left(\Gamma_{2}\right)^{8}\right)\left(s^{3}\right)^{12}\left(s^{2}\right)^{6}+2\left(19500\left(\Gamma_{1}\right)^{8}+90722\left(\Gamma_{2}\right)\left(\Gamma_{1}\right)^{7}\right.$ $+340099\left(\Gamma_{2}\right)^{2}\left(\Gamma_{1}\right)^{6}+892894\left(\Gamma_{2}\right)^{3}\left(\Gamma_{1}\right)^{5}+1307489\left(\Gamma_{2}\right)^{4}\left(\Gamma_{1}\right)^{4}+1063494\left(\Gamma_{2}\right)^{5}\left(\Gamma_{1}\right)^{3}$ $\left.+517084\left(\Gamma_{2}\right)^{6}\left(\Gamma_{1}\right)^{2}+177192\left(\Gamma_{2}\right)^{7}\left(\Gamma_{1}\right)+40140\left(\Gamma_{2}\right)^{8}\right)\left(s^{3}\right)^{14}\left(s^{2}\right)^{4}-\left(13650\left(\Gamma_{1}\right)^{8}\right.$ $+50046\left(\Gamma_{2}\right)\left(\Gamma_{1}\right)^{7}+347402\left(\Gamma_{2}\right)^{2}\left(\Gamma_{1}\right)^{6}+1239806\left(\Gamma_{2}\right)^{3}\left(\Gamma_{1}\right)^{5}+1896415\left(\Gamma_{2}\right)^{4}\left(\Gamma_{1}\right)^{4}$ $\left.+1340896\left(\Gamma_{2}\right)^{5}\left(\Gamma_{1}\right)^{3}+470030\left(\Gamma_{2}\right)^{6}\left(\Gamma_{1}\right)^{2}+144000\left(\Gamma_{2}\right)^{7}\left(\Gamma_{1}\right)+47250\left(\Gamma_{2}\right)^{8}\right)\left(s^{3}\right)^{16}\left(s^{2}\right)^{2}$ $+\left(1950\left(\Gamma_{1}\right)^{8}+4314\left(\Gamma_{2}\right)\left(\Gamma_{1}\right)^{7}+86126\left(\Gamma_{2}\right)^{2}\left(\Gamma_{1}\right)^{6}+365668\left(\Gamma_{2}\right)^{3}\left(\Gamma_{1}\right)^{5}+532355\left(\Gamma_{2}\right)^{4}\left(\Gamma_{1}\right)^{4}\right.$ $\left.\left.+248364\left(\Gamma_{2}\right)^{5}\left(\Gamma_{1}\right)^{3}-32060\left(\Gamma_{2}\right)^{6}\left(\Gamma_{1}\right)^{2}-5720\left(\Gamma_{2}\right)^{7}\left(\Gamma_{1}\right)+24255\left(\Gamma_{2}\right)^{8}\right)\left(s^{3}\right)^{18}\right)\left(s^{1}\right)^{6}$ $+2\left(\left(\Gamma_{1}\right)^{2}\left(3018\left(\Gamma_{1}\right)^{6}+28340\left(\Gamma_{2}\right)\left(\Gamma_{1}\right)^{5}+163356\left(\Gamma_{2}\right)^{2}\left(\Gamma_{1}\right)^{4}+247924\left(\Gamma_{2}\right)^{3}\left(\Gamma_{1}\right)^{3}\right.\right.$ $\left.+41851\left(\Gamma_{2}\right)^{4}\left(\Gamma_{1}\right)^{2}-77152\left(\Gamma_{2}\right)^{5}\left(\Gamma_{1}\right)+5440\left(\Gamma_{2}\right)^{6}\right)\left(s^{2}\right)^{20}-4\left(\Gamma_{1}\right)\left(5745\left(\Gamma_{1}\right)^{7}\right.$ $+40384\left(\Gamma_{2}\right)\left(\Gamma_{1}\right)^{6}+197888\left(\Gamma_{2}\right)^{2}\left(\Gamma_{1}\right)^{5}+298535\left(\Gamma_{2}\right)^{3}\left(\Gamma_{1}\right)^{4}+101802\left(\Gamma_{2}\right)^{4}\left(\Gamma_{1}\right)^{3}$ $\left.-52098\left(\Gamma_{2}\right)^{5}\left(\Gamma_{1}\right)^{2}-7208\left(\Gamma_{2}\right)^{6}\left(\Gamma_{1}\right)+381\left(\Gamma_{2}\right)^{7}\right)\left(s^{3}\right)^{2}\left(s^{2}\right)^{18}+\left(78210\left(\Gamma_{1}\right)^{8}\right.$ $+388908\left(\Gamma_{2}\right)\left(\Gamma_{1}\right)^{7}+1511656\left(\Gamma_{2}\right)^{2}\left(\Gamma_{1}\right)^{6}+2218296\left(\Gamma_{2}\right)^{3}\left(\Gamma_{1}\right)^{5}+1041151\left(\Gamma_{2}\right)^{4}\left(\Gamma_{1}\right)^{4}$ $\left.-81588\left(\Gamma_{2}\right)^{5}\left(\Gamma_{1}\right)^{3}-27182\left(\Gamma_{2}\right)^{6}\left(\Gamma_{1}\right)^{2}+12\left(\Gamma_{2}\right)^{7}\left(\Gamma_{1}\right)+3\left(\Gamma_{2}\right)^{8}\right)\left(s^{3}\right)^{4}\left(s^{2}\right)^{16}-4\left(40140\left(\Gamma_{1}\right)^{8}\right.$ $+143928\left(\Gamma_{2}\right)\left(\Gamma_{1}\right)^{7}+400660\left(\Gamma_{2}\right)^{2}\left(\Gamma_{1}\right)^{6}+565818\left(\Gamma_{2}\right)^{3}\left(\Gamma_{1}\right)^{5}+354359\left(\Gamma_{2}\right)^{4}\left(\Gamma_{1}\right)^{4}$ $\left.+89922\left(\Gamma_{2}\right)^{5}\left(\Gamma_{1}\right)^{3}+30559\left(\Gamma_{2}\right)^{6}\left(\Gamma_{1}\right)^{2}+6560\left(\Gamma_{2}\right)^{7}\left(\Gamma_{1}\right)+10\left(\Gamma_{2}\right)^{8}\right)\left(s^{3}\right)^{6}\left(s^{2}\right)^{14}$ $+2\left(115290\left(\Gamma_{1}\right)^{8}+379092\left(\Gamma_{2}\right)\left(\Gamma_{1}\right)^{7}+832300\left(\Gamma_{2}\right)^{2}\left(\Gamma_{1}\right)^{6}+1200768\left(\Gamma_{2}\right)^{3}\left(\Gamma_{1}\right)^{5}\right.$ $+1042919\left(\Gamma_{2}\right)^{4}\left(\Gamma_{1}\right)^{4}+542498\left(\Gamma_{2}\right)^{5}\left(\Gamma_{1}\right)^{3}+193294\left(\Gamma_{2}\right)^{6}\left(\Gamma_{1}\right)^{2}+35538\left(\Gamma_{2}\right)^{7}\left(\Gamma_{1}\right)$ $\left.+335\left(\Gamma_{2}\right)^{8}\right)\left(s^{3}\right)^{8}\left(s^{2}\right)^{12}-4\left(64134\left(\Gamma_{1}\right)^{8}+256536\left(\Gamma_{2}\right)\left(\Gamma_{1}\right)^{7}+603764\left(\Gamma_{2}\right)^{2}\left(\Gamma_{1}\right)^{6}\right.$ $+913416\left(\Gamma_{2}\right)^{3}\left(\Gamma_{1}\right)^{5}+865073\left(\Gamma_{2}\right)^{4}\left(\Gamma_{1}\right)^{4}+507078\left(\Gamma_{2}\right)^{5}\left(\Gamma_{1}\right)^{3}+187670\left(\Gamma_{2}\right)^{6}\left(\Gamma_{1}\right)^{2}$ $\left.+43611\left(\Gamma_{2}\right)^{7}\left(\Gamma_{1}\right)+2745\left(\Gamma_{2}\right)^{8}\right)\left(s^{3}\right)^{10}\left(s^{2}\right)^{10}+2\left(115290\left(\Gamma_{1}\right)^{8}+543228\left(\Gamma_{2}\right)\left(\Gamma_{1}\right)^{7}\right.$ $+1406776\left(\Gamma_{2}\right)^{2}\left(\Gamma_{1}\right)^{6}+2288340\left(\Gamma_{2}\right)^{3}\left(\Gamma_{1}\right)^{5}+2325659\left(\Gamma_{2}\right)^{4}\left(\Gamma_{1}\right)^{4}+1455518\left(\Gamma_{2}\right)^{5}\left(\Gamma_{1}\right)^{3}$ $\left.+567322\left(\Gamma_{2}\right)^{6}\left(\Gamma_{1}\right)^{2}+153868\left(\Gamma_{2}\right)^{7}\left(\Gamma_{1}\right)+26242\left(\Gamma_{2}\right)^{8}\right)\left(s^{3}\right)^{12}\left(s^{2}\right)^{8}-4\left(40140\left(\Gamma_{1}\right)^{8}\right.$ $+177192\left(\Gamma_{2}\right)\left(\Gamma_{1}\right)^{7}+517084\left(\Gamma_{2}\right)^{2}\left(\Gamma_{1}\right)^{6}+1063494\left(\Gamma_{2}\right)^{3}\left(\Gamma_{1}\right)^{5}+1307489\left(\Gamma_{2}\right)^{4}\left(\Gamma_{1}\right)^{4}$ $\left.+892894\left(\Gamma_{2}\right)^{5}\left(\Gamma_{1}\right)^{3}+340099\left(\Gamma_{2}\right)^{6}\left(\Gamma_{1}\right)^{2}+90722\left(\Gamma_{2}\right)^{7}\left(\Gamma_{1}\right)+19500\left(\Gamma_{2}\right)^{8}\right)\left(s^{3}\right)^{14}\left(s^{2}\right)^{6}$ $+\left(78210\left(\Gamma_{1}\right)^{8}+236772\left(\Gamma_{2}\right)\left(\Gamma_{1}\right)^{7}+979180\left(\Gamma_{2}\right)^{2}\left(\Gamma_{1}\right)^{6}+3064332\left(\Gamma_{2}\right)^{3}\left(\Gamma_{1}\right)^{5}\right.$ $+4487431\left(\Gamma_{2}\right)^{4}\left(\Gamma_{1}\right)^{4}+3064332\left(\Gamma_{2}\right)^{5}\left(\Gamma_{1}\right)^{3}+979180\left(\Gamma_{2}\right)^{6}\left(\Gamma_{1}\right)^{2}+236772\left(\Gamma_{2}\right)^{7}\left(\Gamma_{1}\right)$ $\left.+78210\left(\Gamma_{2}\right)^{8}\right)\left(s^{3}\right)^{16}\left(s^{2}\right)^{4}-4\left(5745\left(\Gamma_{1}\right)^{8}+5576\left(\Gamma_{2}\right)\left(\Gamma_{1}\right)^{7}+76060\left(\Gamma_{2}\right)^{2}\left(\Gamma_{1}\right)^{6}\right.$ $+362449\left(\Gamma_{2}\right)^{3}\left(\Gamma_{1}\right)^{5}+566157\left(\Gamma_{2}\right)^{4}\left(\Gamma_{1}\right)^{4}+339996\left(\Gamma_{2}\right)^{5}\left(\Gamma_{1}\right)^{3}+55664\left(\Gamma_{2}\right)^{6}\left(\Gamma_{1}\right)^{2}$ $\left.+6630\left(\Gamma_{2}\right)^{7}\left(\Gamma_{1}\right)+11025\left(\Gamma_{2}\right)^{8}\right)\left(s^{3}\right)^{18}\left(s^{2}\right)^{2}+\left(\left(\Gamma_{1}\right)+\left(\Gamma_{2}\right)\right)^{2}\left(3018\left(\Gamma_{1}\right)^{6}-10232\left(\Gamma_{2}\right)\left(\Gamma_{1}\right)^{5}\right.$ $+66926\left(\Gamma_{2}\right)^{2}\left(\Gamma_{1}\right)^{4}+182460\left(\Gamma_{2}\right)^{3}\left(\Gamma_{1}\right)^{3}+40085\left(\Gamma_{2}\right)^{4}\left(\Gamma_{1}\right)^{2}-53086\left(\Gamma_{2}\right)^{5}\left(\Gamma_{1}\right)$ $\left.\left.+14553\left(\Gamma_{2}\right)^{6}\right)\left(s^{3}\right)^{20}\right)\left(s^{1}\right)^{4}-4\left(\left(s^{2}\right)^{2}-\left(s^{3}\right)^{2}\right)^{2}\left(\left(\Gamma_{1}\right)^{3}\left(630\left(\Gamma_{1}\right)^{5}+7152\left(\Gamma_{2}\right)\left(\Gamma_{1}\right)^{4}\right.\right.$ $\left.+33810\left(\Gamma_{2}\right)^{2}\left(\Gamma_{1}\right)^{3}+51328\left(\Gamma_{2}\right)^{3}\left(\Gamma_{1}\right)^{2}+18667\left(\Gamma_{2}\right)^{4}\left(\Gamma_{1}\right)-6696\left(\Gamma_{2}\right)^{5}\right)\left(s^{2}\right)^{18}$ $-2\left(\Gamma_{1}\right)^{2}\left(2205\left(\Gamma_{1}\right)^{6}+19674\left(\Gamma_{2}\right)\left(\Gamma_{1}\right)^{5}+82274\left(\Gamma_{2}\right)^{2}\left(\Gamma_{1}\right)^{4}+124468\left(\Gamma_{2}\right)^{3}\left(\Gamma_{1}\right)^{3}\right.$ $\left.+62431\left(\Gamma_{2}\right)^{4}\left(\Gamma_{1}\right)^{2}-1464\left(\Gamma_{2}\right)^{5}\left(\Gamma_{1}\right)-2720\left(\Gamma_{2}\right)^{6}\right)\left(s^{3}\right)^{2}\left(s^{2}\right)^{16}+\left(\Gamma_{1}\right)\left(12600\left(\Gamma_{1}\right)^{7}\right.$ $+77292\left(\Gamma_{2}\right)\left(\Gamma_{1}\right)^{6}+273002\left(\Gamma_{2}\right)^{2}\left(\Gamma_{1}\right)^{5}+395116\left(\Gamma_{2}\right)^{3}\left(\Gamma_{1}\right)^{4}+213283\left(\Gamma_{2}\right)^{4}\left(\Gamma_{1}\right)^{3}$ $\left.+14250\left(\Gamma_{2}\right)^{5}\left(\Gamma_{1}\right)^{2}-8870\left(\Gamma_{2}\right)^{6}\left(\Gamma_{1}\right)-762\left(\Gamma_{2}\right)^{7}\right)\left(s^{3}\right)^{4}\left(s^{2}\right)^{14}+\left(-17640\left(\Gamma_{1}\right)^{8}\right.$ 
$-40068\left(\Gamma_{2}\right)\left(\Gamma_{1}\right)^{7}-74478\left(\Gamma_{2}\right)^{2}\left(\Gamma_{1}\right)^{6}-62116\left(\Gamma_{2}\right)^{3}\left(\Gamma_{1}\right)^{5}+41543\left(\Gamma_{2}\right)^{4}\left(\Gamma_{1}\right)^{4}$ $\left.+82078\left(\Gamma_{2}\right)^{5}\left(\Gamma_{1}\right)^{3}+29856\left(\Gamma_{2}\right)^{6}\left(\Gamma_{1}\right)^{2}+1776\left(\Gamma_{2}\right)^{7}\left(\Gamma_{1}\right)+\left(\Gamma_{2}\right)^{8}\right)\left(s^{3}\right)^{6}\left(s^{2}\right)^{12}$ $+\left(8820\left(\Gamma_{1}\right)^{8}-80388\left(\Gamma_{2}\right)\left(\Gamma_{1}\right)^{7}-331546\left(\Gamma_{2}\right)^{2}\left(\Gamma_{1}\right)^{6}-532980\left(\Gamma_{2}\right)^{3}\left(\Gamma_{1}\right)^{5}-483201\left(\Gamma_{2}\right)^{4}\left(\Gamma_{1}\right)^{4}\right.$ $\left.-262912\left(\Gamma_{2}\right)^{5}\left(\Gamma_{1}\right)^{3}-79264\left(\Gamma_{2}\right)^{6}\left(\Gamma_{1}\right)^{2}-9256\left(\Gamma_{2}\right)^{7}\left(\Gamma_{1}\right)-28\left(\Gamma_{2}\right)^{8}\right)\left(s^{3}\right)^{8}\left(s^{2}\right)^{10}+\left(8820\left(\Gamma_{1}\right)^{8}\right.$ $+150948\left(\Gamma_{2}\right)\left(\Gamma_{1}\right)^{7}+478130\left(\Gamma_{2}\right)^{2}\left(\Gamma_{1}\right)^{6}+725772\left(\Gamma_{2}\right)^{3}\left(\Gamma_{1}\right)^{5}+639489\left(\Gamma_{2}\right)^{4}\left(\Gamma_{1}\right)^{4}$ $\left.+336488\left(\Gamma_{2}\right)^{5}\left(\Gamma_{1}\right)^{3}+101984\left(\Gamma_{2}\right)^{6}\left(\Gamma_{1}\right)^{2}+15560\left(\Gamma_{2}\right)^{7}\left(\Gamma_{1}\right)+317\left(\Gamma_{2}\right)^{8}\right)\left(s^{3}\right)^{10}\left(s^{2}\right)^{8}$ $-\left(17640\left(\Gamma_{1}\right)^{8}+101052\left(\Gamma_{2}\right)\left(\Gamma_{1}\right)^{7}+287922\left(\Gamma_{2}\right)^{2}\left(\Gamma_{1}\right)^{6}+531164\left(\Gamma_{2}\right)^{3}\left(\Gamma_{1}\right)^{5}\right.$ $+597467\left(\Gamma_{2}\right)^{4}\left(\Gamma_{1}\right)^{4}+369766\left(\Gamma_{2}\right)^{5}\left(\Gamma_{1}\right)^{3}+115622\left(\Gamma_{2}\right)^{6}\left(\Gamma_{1}\right)^{2}+19058\left(\Gamma_{2}\right)^{7}\left(\Gamma_{1}\right)$ $\left.+2388\left(\Gamma_{2}\right)^{8}\right)\left(s^{3}\right)^{12}\left(s^{2}\right)^{6}+\left(12600\left(\Gamma_{1}\right)^{8}+23508\left(\Gamma_{2}\right)\left(\Gamma_{1}\right)^{7}+84758\left(\Gamma_{2}\right)^{2}\left(\Gamma_{1}\right)^{6}\right.$ $+325364\left(\Gamma_{2}\right)^{3}\left(\Gamma_{1}\right)^{5}+509513\left(\Gamma_{2}\right)^{4}\left(\Gamma_{1}\right)^{4}+348142\left(\Gamma_{2}\right)^{5}\left(\Gamma_{1}\right)^{3}+101616\left(\Gamma_{2}\right)^{6}\left(\Gamma_{1}\right)^{2}$ $\left.+15592\left(\Gamma_{2}\right)^{7}\left(\Gamma_{1}\right)+4119\left(\Gamma_{2}\right)^{8}\right)\left(s^{3}\right)^{14}\left(s^{2}\right)^{4}-2\left(\left(\Gamma_{1}\right)+\left(\Gamma_{2}\right)\right)^{2}\left(2205\left(\Gamma_{1}\right)^{6}-6444\left(\Gamma_{2}\right)\left(\Gamma_{1}\right)^{5}\right.$ $+16979\left(\Gamma_{2}\right)^{2}\left(\Gamma_{1}\right)^{4}+51988\left(\Gamma_{2}\right)^{3}\left(\Gamma_{1}\right)^{3}+19006\left(\Gamma_{2}\right)^{4}\left(\Gamma_{1}\right)^{2}-3122\left(\Gamma_{2}\right)^{5}\left(\Gamma_{1}\right)$ $\left.+1512\left(\Gamma_{2}\right)^{6}\right)\left(s^{3}\right)^{16}\left(s^{2}\right)^{2}+\left(\left(\Gamma_{1}\right)+\left(\Gamma_{2}\right)\right)^{3}\left(630\left(\Gamma_{1}\right)^{5}-4002\left(\Gamma_{2}\right)\left(\Gamma_{1}\right)^{4}+11502\left(\Gamma_{2}\right)^{2}\left(\Gamma_{1}\right)^{3}\right.$ $\left.\left.+13490\left(\Gamma_{2}\right)^{3}\left(\Gamma_{1}\right)^{2}-8017\left(\Gamma_{2}\right)^{4}\left(\Gamma_{1}\right)+1323\left(\Gamma_{2}\right)^{5}\right)\left(s^{3}\right)^{18}\right)\left(s^{1}\right)^{2}$

$+\left(\left(s^{2}\right)^{2}-\left(s^{3}\right)^{2}\right)^{4}\left(\left(\Gamma_{1}\right)^{4}\left(441\left(\Gamma_{1}\right)^{4}+5500\left(\Gamma_{2}\right)\left(\Gamma_{1}\right)^{3}+23662\left(\Gamma_{2}\right)^{2}\left(\Gamma_{1}\right)^{2}+36324\left(\Gamma_{2}\right)^{3}\left(\Gamma_{1}\right)\right.\right.$ $\left.+18162\left(\Gamma_{2}\right)^{4}\right)\left(s^{2}\right)^{16}-4\left(\Gamma_{1}\right)^{3}\left(882\left(\Gamma_{1}\right)^{5}+9132\left(\Gamma_{2}\right)\left(\Gamma_{1}\right)^{4}+35126\left(\Gamma_{2}\right)^{2}\left(\Gamma_{1}\right)^{3}+53976\left(\Gamma_{2}\right)^{3}\left(\Gamma_{1}\right)^{2}\right.$ $\left.+33985\left(\Gamma_{2}\right)^{4}\left(\Gamma_{1}\right)+6696\left(\Gamma_{2}\right)^{5}\right)\left(s^{3}\right)^{2}\left(s^{2}\right)^{14}+2\left(\Gamma_{1}\right)^{2}\left(6174\left(\Gamma_{1}\right)^{6}+50848\left(\Gamma_{2}\right)\left(\Gamma_{1}\right)^{5}\right.$ $+171816\left(\Gamma_{2}\right)^{2}\left(\Gamma_{1}\right)^{4}+259020\left(\Gamma_{2}\right)^{3}\left(\Gamma_{1}\right)^{3}+182845\left(\Gamma_{2}\right)^{4}\left(\Gamma_{1}\right)^{2}+56224\left(\Gamma_{2}\right)^{5}\left(\Gamma_{1}\right)$ $\left.+5440\left(\Gamma_{2}\right)^{6}\right)\left(s^{3}\right)^{4}\left(s^{2}\right)^{12}-4\left(\Gamma_{1}\right)\left(6174\left(\Gamma_{1}\right)^{7}+37772\left(\Gamma_{2}\right)\left(\Gamma_{1}\right)^{6}+109070\left(\Gamma_{2}\right)^{2}\left(\Gamma_{1}\right)^{5}\right.$ $+159456\left(\Gamma_{2}\right)^{3}\left(\Gamma_{1}\right)^{4}+119923\left(\Gamma_{2}\right)^{4}\left(\Gamma_{1}\right)^{3}+44768\left(\Gamma_{2}\right)^{5}\left(\Gamma_{1}\right)^{2}+7092\left(\Gamma_{2}\right)^{6}\left(\Gamma_{1}\right)$ $\left.+254\left(\Gamma_{2}\right)^{7}\right)\left(s^{3}\right)^{6}\left(s^{2}\right)^{10}+\left(30870\left(\Gamma_{1}\right)^{8}+123480\left(\Gamma_{2}\right)\left(\Gamma_{1}\right)^{7}+288220\left(\Gamma_{2}\right)^{2}\left(\Gamma_{1}\right)^{6}\right.$ $+432480\left(\Gamma_{2}\right)^{3}\left(\Gamma_{1}\right)^{5}+374840\left(\Gamma_{2}\right)^{4}\left(\Gamma_{1}\right)^{4}+172940\left(\Gamma_{2}\right)^{5}\left(\Gamma_{1}\right)^{3}+37502\left(\Gamma_{2}\right)^{6}\left(\Gamma_{1}\right)^{2}$ $\left.+2532\left(\Gamma_{2}\right)^{7}\left(\Gamma_{1}\right)+\left(\Gamma_{2}\right)^{8}\right)\left(s^{3}\right)^{8}\left(s^{2}\right)^{8}-4\left(6174\left(\Gamma_{1}\right)^{8}+11620\left(\Gamma_{2}\right)\left(\Gamma_{1}\right)^{7}+17538\left(\Gamma_{2}\right)^{2}\left(\Gamma_{1}\right)^{6}\right.$ $+47496\left(\Gamma_{2}\right)^{3}\left(\Gamma_{1}\right)^{5}+68853\left(\Gamma_{2}\right)^{4}\left(\Gamma_{1}\right)^{4}+45488\left(\Gamma_{2}\right)^{5}\left(\Gamma_{1}\right)^{3}+13476\left(\Gamma_{2}\right)^{6}\left(\Gamma_{1}\right)^{2}$ $\left.+1446\left(\Gamma_{2}\right)^{7}\left(\Gamma_{1}\right)+9\left(\Gamma_{2}\right)^{8}\right)\left(s^{3}\right)^{10}\left(s^{2}\right)^{6}+2\left(\left(\Gamma_{1}\right)+\left(\Gamma_{2}\right)\right)^{2}\left(6174\left(\Gamma_{1}\right)^{6}-13804\left(\Gamma_{2}\right)\left(\Gamma_{1}\right)^{5}\right.$ $+10186\left(\Gamma_{2}\right)^{2}\left(\Gamma_{1}\right)^{4}+43244\left(\Gamma_{2}\right)^{3}\left(\Gamma_{1}\right)^{3}+20811\left(\Gamma_{2}\right)^{4}\left(\Gamma_{1}\right)^{2}+2474\left(\Gamma_{2}\right)^{5}\left(\Gamma_{1}\right)$ $\left.+183\left(\Gamma_{2}\right)^{6}\right)\left(s^{3}\right)^{12}\left(s^{2}\right)^{4}-4\left(\left(\Gamma_{1}\right)+\left(\Gamma_{2}\right)\right)^{3}\left(882\left(\Gamma_{1}\right)^{5}-4722\left(\Gamma_{2}\right)\left(\Gamma_{1}\right)^{4}+7418\left(\Gamma_{2}\right)^{2}\left(\Gamma_{1}\right)^{3}\right.$ $\left.+5430\left(\Gamma_{2}\right)^{3}\left(\Gamma_{1}\right)^{2}-707\left(\Gamma_{2}\right)^{4}\left(\Gamma_{1}\right)+189\left(\Gamma_{2}\right)^{5}\right)\left(s^{3}\right)^{14}\left(s^{2}\right)^{2}+\left(\left(\Gamma_{1}\right)+\left(\Gamma_{2}\right)\right)^{4}\left(441\left(\Gamma_{1}\right)^{4}\right.$ $\left.\left.-3736\left(\Gamma_{2}\right)\left(\Gamma_{1}\right)^{3}+9808\left(\Gamma_{2}\right)^{2}\left(\Gamma_{1}\right)^{2}-3736\left(\Gamma_{2}\right)^{3}\left(\Gamma_{1}\right)+441\left(\Gamma_{2}\right)^{4}\right)\left(s^{3}\right)^{16}\right)$, $w\left(s^{1}, s^{2}, s^{3}\right)=21\left(\Gamma_{1}\right)^{2}\left(\Gamma_{2}\right)^{2}\left(20\left(\Gamma_{2}\right)^{3}\left(117\left(\Gamma_{1}\right)^{2}+117\left(\Gamma_{2}\right)\left(\Gamma_{1}\right)+20\left(\Gamma_{2}\right)^{2}\right)\left(\left(\Gamma_{1}\right)\left(s^{2}\right)^{2}\right.\right.$ $\left.-\left(\left(\Gamma_{1}\right)+\left(\Gamma_{2}\right)\right)\left(s^{3}\right)^{2}\right)\left(s^{1}\right)^{22}+\left(\Gamma_{2}\right)^{2}\left(\left(3501\left(\Gamma_{1}\right)^{4}-7078\left(\Gamma_{2}\right)\left(\Gamma_{1}\right)^{3}-3139\left(\Gamma_{2}\right)^{2}\left(\Gamma_{1}\right)^{2}\right.\right.$ $\left.+4640\left(\Gamma_{2}\right)^{3}\left(\Gamma_{1}\right)+1200\left(\Gamma_{2}\right)^{4}\right)\left(s^{2}\right)^{4}-6\left(\Gamma_{1}\right)\left(1167\left(\Gamma_{1}\right)^{3}+2334\left(\Gamma_{2}\right)\left(\Gamma_{1}\right)^{2}+337\left(\Gamma_{2}\right)^{2}\left(\Gamma_{1}\right)\right.$ $\left.-830\left(\Gamma_{2}\right)^{3}\right)\left(s^{3}\right)^{2}\left(s^{2}\right)^{2}+\left(3501\left(\Gamma_{1}\right)^{4}+21082\left(\Gamma_{2}\right)\left(\Gamma_{1}\right)^{3}+39101\left(\Gamma_{2}\right)^{2}\left(\Gamma_{1}\right)^{2}\right.$ $\left.\left.+24320\left(\Gamma_{2}\right)^{3}\left(\Gamma_{1}\right)+4000\left(\Gamma_{2}\right)^{4}\right)\left(s^{3}\right)^{4}\right)\left(s^{1}\right)^{20}-4\left(\Gamma_{2}\right)\left(\left(-490\left(\Gamma_{1}\right)^{5}+1737\left(\Gamma_{2}\right)\left(\Gamma_{1}\right)^{4}\right.\right.$ $\left.-6981\left(\Gamma_{2}\right)^{2}\left(\Gamma_{1}\right)^{3}-938\left(\Gamma_{2}\right)^{3}\left(\Gamma_{1}\right)^{2}+4570\left(\Gamma_{2}\right)^{4}\left(\Gamma_{1}\right)+800\left(\Gamma_{2}\right)^{5}\right)\left(s^{2}\right)^{6}+\left(1470\left(\Gamma_{1}\right)^{5}\right.$ $\left.+713\left(\Gamma_{2}\right)\left(\Gamma_{1}\right)^{4}-6809\left(\Gamma_{2}\right)^{2}\left(\Gamma_{1}\right)^{3}+2108\left(\Gamma_{2}\right)^{3}\left(\Gamma_{1}\right)^{2}+8960\left(\Gamma_{2}\right)^{4}\left(\Gamma_{1}\right)+800\left(\Gamma_{2}\right)^{5}\right)\left(s^{3}\right)^{2}\left(s^{2}\right)^{4}$ $+\left(\Gamma_{1}\right)\left(-1470\left(\Gamma_{1}\right)^{4}-6637\left(\Gamma_{2}\right)\left(\Gamma_{1}\right)^{3}-5039\left(\Gamma_{2}\right)^{2}\left(\Gamma_{1}\right)^{2}+12113\left(\Gamma_{2}\right)^{3}\left(\Gamma_{1}\right)\right.$ $\left.+11185\left(\Gamma_{2}\right)^{4}\right)\left(s^{3}\right)^{4}\left(s^{2}\right)^{2}+\left(490\left(\Gamma_{1}\right)^{5}+4187\left(\Gamma_{2}\right)\left(\Gamma_{1}\right)^{4}+18829\left(\Gamma_{2}\right)^{2}\left(\Gamma_{1}\right)^{3}\right.$ $\left.\left.+35327\left(\Gamma_{2}\right)^{3}\left(\Gamma_{1}\right)^{2}+23895\left(\Gamma_{2}\right)^{4}\left(\Gamma_{1}\right)+4500\left(\Gamma_{2}\right)^{5}\right)\left(s^{3}\right)^{6}\right)\left(s^{1}\right)^{18}+2\left(2\left(100\left(\Gamma_{1}\right)^{6}\right.\right.$ $-380\left(\Gamma_{2}\right)\left(\Gamma_{1}\right)^{5}+1767\left(\Gamma_{2}\right)^{2}\left(\Gamma_{1}\right)^{4}-19746\left(\Gamma_{2}\right)^{3}\left(\Gamma_{1}\right)^{3}-4033\left(\Gamma_{2}\right)^{4}\left(\Gamma_{1}\right)^{2}$
$\left.+6260\left(\Gamma_{2}\right)^{5}\left(\Gamma_{1}\right)+300\left(\Gamma_{2}\right)^{6}\right)\left(s^{2}\right)^{8}+5\left(-160\left(\Gamma_{1}\right)^{6}+64\left(\Gamma_{2}\right)\left(\Gamma_{1}\right)^{5}+1607\left(\Gamma_{2}\right)^{2}\left(\Gamma_{1}\right)^{4}\right.$ $\left.-3550\left(\Gamma_{2}\right)^{3}\left(\Gamma_{1}\right)^{3}+5047\left(\Gamma_{2}\right)^{4}\left(\Gamma_{1}\right)^{2}+11100\left(\Gamma_{2}\right)^{5}\left(\Gamma_{1}\right)+1120\left(\Gamma_{2}\right)^{6}\right)\left(s^{3}\right)^{2}\left(s^{2}\right)^{6}$ $+2\left(600\left(\Gamma_{1}\right)^{6}+1800\left(\Gamma_{2}\right)\left(\Gamma_{1}\right)^{5}-8169\left(\Gamma_{2}\right)^{2}\left(\Gamma_{1}\right)^{4}-19338\left(\Gamma_{2}\right)^{3}\left(\Gamma_{1}\right)^{3}+7211\left(\Gamma_{2}\right)^{4}\left(\Gamma_{1}\right)^{2}\right.$ $\left.+17180\left(\Gamma_{2}\right)^{5}\left(\Gamma_{1}\right)-3700\left(\Gamma_{2}\right)^{6}\right)\left(s^{3}\right)^{4}\left(s^{2}\right)^{4}-5\left(\Gamma_{1}\right)\left(160\left(\Gamma_{1}\right)^{5}+1024\left(\Gamma_{2}\right)\left(\Gamma_{1}\right)^{4}\right.$ $\left.+1113\left(\Gamma_{2}\right)^{2}\left(\Gamma_{1}\right)^{3}-6138\left(\Gamma_{2}\right)^{3}\left(\Gamma_{1}\right)^{2}-22299\left(\Gamma_{2}\right)^{4}\left(\Gamma_{1}\right)-14792\left(\Gamma_{2}\right)^{5}\right)\left(s^{3}\right)^{6}\left(s^{2}\right)^{2}$ $+2\left(100\left(\Gamma_{1}\right)^{6}+980\left(\Gamma_{2}\right)\left(\Gamma_{1}\right)^{5}+5167\left(\Gamma_{2}\right)^{2}\left(\Gamma_{1}\right)^{4}+32614\left(\Gamma_{2}\right)^{3}\left(\Gamma_{1}\right)^{3}+71107\left(\Gamma_{2}\right)^{4}\left(\Gamma_{1}\right)^{2}\right.$ $\left.\left.+54480\left(\Gamma_{2}\right)^{5}\left(\Gamma_{1}\right)+12000\left(\Gamma_{2}\right)^{6}\right)\left(s^{3}\right)^{8}\right)\left(s^{1}\right)^{16}-2\left(\Gamma_{2}\right)\left(4\left(1155\left(\Gamma_{1}\right)^{5}+1777\left(\Gamma_{2}\right)\left(\Gamma_{1}\right)^{4}\right.\right.$ $\left.-14921\left(\Gamma_{2}\right)^{2}\left(\Gamma_{1}\right)^{3}-1868\left(\Gamma_{2}\right)^{3}\left(\Gamma_{1}\right)^{2}+2775\left(\Gamma_{2}\right)^{4}\left(\Gamma_{1}\right)-400\left(\Gamma_{2}\right)^{5}\right)\left(s^{2}\right)^{10}-3\left(5280\left(\Gamma_{1}\right)^{5}\right.$ $+3867\left(\Gamma_{2}\right)\left(\Gamma_{1}\right)^{4}+19704\left(\Gamma_{2}\right)^{2}\left(\Gamma_{1}\right)^{3}+887\left(\Gamma_{2}\right)^{3}\left(\Gamma_{1}\right)^{2}-20830\left(\Gamma_{2}\right)^{4}\left(\Gamma_{1}\right)$ $\left.-600\left(\Gamma_{2}\right)^{5}\right)\left(s^{3}\right)^{2}\left(s^{2}\right)^{8}+\left(24420\left(\Gamma_{1}\right)^{5}+37493\left(\Gamma_{2}\right)\left(\Gamma_{1}\right)^{4}+62926\left(\Gamma_{2}\right)^{2}\left(\Gamma_{1}\right)^{3}\right.$ $\left.+127693\left(\Gamma_{2}\right)^{3}\left(\Gamma_{1}\right)^{2}+128240\left(\Gamma_{2}\right)^{4}\left(\Gamma_{1}\right)+4000\left(\Gamma_{2}\right)^{5}\right)\left(s^{3}\right)^{4}\left(s^{2}\right)^{6}-\left(24420\left(\Gamma_{1}\right)^{5}\right.$ $+84607\left(\Gamma_{2}\right)\left(\Gamma_{1}\right)^{4}+157154\left(\Gamma_{2}\right)^{2}\left(\Gamma_{1}\right)^{3}+80327\left(\Gamma_{2}\right)^{3}\left(\Gamma_{1}\right)^{2}+33760\left(\Gamma_{2}\right)^{4}\left(\Gamma_{1}\right)$ $\left.+46400\left(\Gamma_{2}\right)^{5}\right)\left(s^{3}\right)^{6}\left(s^{2}\right)^{4}+3\left(\Gamma_{1}\right)\left(5280\left(\Gamma_{1}\right)^{4}+22533\left(\Gamma_{2}\right)\left(\Gamma_{1}\right)^{3}+57036\left(\Gamma_{2}\right)^{2}\left(\Gamma_{1}\right)^{2}\right.$ $\left.+87823\left(\Gamma_{2}\right)^{3}\left(\Gamma_{1}\right)+47440\left(\Gamma_{2}\right)^{4}\right)\left(s^{3}\right)^{8}\left(s^{2}\right)^{2}+4\left(-1155\left(\Gamma_{1}\right)^{5}-3998\left(\Gamma_{2}\right)\left(\Gamma_{1}\right)^{4}\right.$ $\left.\left.+10479\left(\Gamma_{2}\right)^{2}\left(\Gamma_{1}\right)^{3}+42007\left(\Gamma_{2}\right)^{3}\left(\Gamma_{1}\right)^{2}+39585\left(\Gamma_{2}\right)^{4}\left(\Gamma_{1}\right)+10500\left(\Gamma_{2}\right)^{5}\right)\left(s^{3}\right)^{10}\right)\left(s^{1}\right)^{14}$ $+\left(-10\left(280\left(\Gamma_{1}\right)^{6}-1920\left(\Gamma_{2}\right)\left(\Gamma_{1}\right)^{5}-883\left(\Gamma_{2}\right)^{2}\left(\Gamma_{1}\right)^{4}+12714\left(\Gamma_{2}\right)^{3}\left(\Gamma_{1}\right)^{3}-883\left(\Gamma_{2}\right)^{4}\left(\Gamma_{1}\right)^{2}\right.\right.$ $\left.-1920\left(\Gamma_{2}\right)^{5}\left(\Gamma_{1}\right)+280\left(\Gamma_{2}\right)^{6}\right)\left(s^{2}\right)^{12}+2\left(6000\left(\Gamma_{1}\right)^{6}-23020\left(\Gamma_{2}\right)\left(\Gamma_{1}\right)^{5}+641\left(\Gamma_{2}\right)^{2}\left(\Gamma_{1}\right)^{4}\right.$ $\left.-46808\left(\Gamma_{2}\right)^{3}\left(\Gamma_{1}\right)^{3}-35679\left(\Gamma_{2}\right)^{4}\left(\Gamma_{1}\right)^{2}+33590\left(\Gamma_{2}\right)^{5}\left(\Gamma_{1}\right)-7200\left(\Gamma_{2}\right)^{6}\right)\left(s^{3}\right)^{2}\left(s^{2}\right)^{10}$ $-5\left(4560\left(\Gamma_{1}\right)^{6}-2576\left(\Gamma_{2}\right)\left(\Gamma_{1}\right)^{5}+18485\left(\Gamma_{2}\right)^{2}\left(\Gamma_{1}\right)^{4}+9994\left(\Gamma_{2}\right)^{3}\left(\Gamma_{1}\right)^{3}-18763\left(\Gamma_{2}\right)^{4}\left(\Gamma_{1}\right)^{2}\right.$ $\left.-46976\left(\Gamma_{2}\right)^{5}\left(\Gamma_{1}\right)-480\left(\Gamma_{2}\right)^{6}\right)\left(s^{3}\right)^{4}\left(s^{2}\right)^{8}+2\left(13600\left(\Gamma_{1}\right)^{6}+40800\left(\Gamma_{2}\right)\left(\Gamma_{1}\right)^{5}\right.$ $+149413\left(\Gamma_{2}\right)^{2}\left(\Gamma_{1}\right)^{4}+230826\left(\Gamma_{2}\right)^{3}\left(\Gamma_{1}\right)^{3}+279653\left(\Gamma_{2}\right)^{4}\left(\Gamma_{1}\right)^{2}+171040\left(\Gamma_{2}\right)^{5}\left(\Gamma_{1}\right)$ $\left.-5600\left(\Gamma_{2}\right)^{6}\right)\left(s^{3}\right)^{6}\left(s^{2}\right)^{6}-5\left(4560\left(\Gamma_{1}\right)^{6}+29936\left(\Gamma_{2}\right)\left(\Gamma_{1}\right)^{5}+99765\left(\Gamma_{2}\right)^{2}\left(\Gamma_{1}\right)^{4}\right.$ $\left.+180906\left(\Gamma_{2}\right)^{3}\left(\Gamma_{1}\right)^{3}+156325\left(\Gamma_{2}\right)^{4}\left(\Gamma_{1}\right)^{2}+93648\left(\Gamma_{2}\right)^{5}\left(\Gamma_{1}\right)+43360\left(\Gamma_{2}\right)^{6}\right)\left(s^{3}\right)^{8}\left(s^{2}\right)^{4}$ $+2\left(\Gamma_{1}\right)\left(6000\left(\Gamma_{1}\right)^{5}+59020\left(\Gamma_{2}\right)\left(\Gamma_{1}\right)^{4}+205741\left(\Gamma_{2}\right)^{2}\left(\Gamma_{1}\right)^{3}+399572\left(\Gamma_{2}\right)^{3}\left(\Gamma_{1}\right)^{2}\right.$ $\left.+428791\left(\Gamma_{2}\right)^{4}\left(\Gamma_{1}\right)+189140\left(\Gamma_{2}\right)^{5}\right)\left(s^{3}\right)^{10}\left(s^{2}\right)^{2}-10\left(280\left(\Gamma_{1}\right)^{6}+3600\left(\Gamma_{2}\right)\left(\Gamma_{1}\right)^{5}\right.$ $+12917\left(\Gamma_{2}\right)^{2}\left(\Gamma_{1}\right)^{4}+8554\left(\Gamma_{2}\right)^{3}\left(\Gamma_{1}\right)^{3}-20923\left(\Gamma_{2}\right)^{4}\left(\Gamma_{1}\right)^{2}-30240\left(\Gamma_{2}\right)^{5}\left(\Gamma_{1}\right)$ $\left.\left.-10080\left(\Gamma_{2}\right)^{6}\right)\left(s^{3}\right)^{12}\right)\left(s^{1}\right)^{12}+2\left(4\left(\Gamma_{1}\right)\left(400\left(\Gamma_{1}\right)^{5}-2775\left(\Gamma_{2}\right)\left(\Gamma_{1}\right)^{4}+1868\left(\Gamma_{2}\right)^{2}\left(\Gamma_{1}\right)^{3}\right.\right.$ $\left.+14921\left(\Gamma_{2}\right)^{3}\left(\Gamma_{1}\right)^{2}-1777\left(\Gamma_{2}\right)^{4}\left(\Gamma_{1}\right)-1155\left(\Gamma_{2}\right)^{5}\right)\left(s^{2}\right)^{14}+\left(-7200\left(\Gamma_{1}\right)^{6}+33590\left(\Gamma_{2}\right)\left(\Gamma_{1}\right)^{5}\right.$ $-35679\left(\Gamma_{2}\right)^{2}\left(\Gamma_{1}\right)^{4}-46808\left(\Gamma_{2}\right)^{3}\left(\Gamma_{1}\right)^{3}+641\left(\Gamma_{2}\right)^{4}\left(\Gamma_{1}\right)^{2}-23020\left(\Gamma_{2}\right)^{5}\left(\Gamma_{1}\right)$ $\left.+6000\left(\Gamma_{2}\right)^{6}\right)\left(s^{3}\right)^{2}\left(s^{2}\right)^{12}+2\left(6000\left(\Gamma_{1}\right)^{6}-20155\left(\Gamma_{2}\right)\left(\Gamma_{1}\right)^{5}+30029\left(\Gamma_{2}\right)^{2}\left(\Gamma_{1}\right)^{4}\right.$ $\left.+41968\left(\Gamma_{2}\right)^{3}\left(\Gamma_{1}\right)^{3}+30029\left(\Gamma_{2}\right)^{4}\left(\Gamma_{1}\right)^{2}-20155\left(\Gamma_{2}\right)^{5}\left(\Gamma_{1}\right)+6000\left(\Gamma_{2}\right)^{6}\right)\left(s^{3}\right)^{4}\left(s^{2}\right)^{10}$ $+\left(-6400\left(\Gamma_{1}\right)^{6}+45080\left(\Gamma_{2}\right)\left(\Gamma_{1}\right)^{5}+36299\left(\Gamma_{2}\right)^{2}\left(\Gamma_{1}\right)^{4}+22828\left(\Gamma_{2}\right)^{3}\left(\Gamma_{1}\right)^{3}-52081\left(\Gamma_{2}\right)^{4}\left(\Gamma_{1}\right)^{2}\right.$ $\left.-93890\left(\Gamma_{2}\right)^{5}\left(\Gamma_{1}\right)+1800\left(\Gamma_{2}\right)^{6}\right)\left(s^{3}\right)^{6}\left(s^{2}\right)^{8}-\left(6400\left(\Gamma_{1}\right)^{6}+83480\left(\Gamma_{2}\right)\left(\Gamma_{1}\right)^{5}\right.$ $+285101\left(\Gamma_{2}\right)^{2}\left(\Gamma_{1}\right)^{4}+456432\left(\Gamma_{2}\right)^{3}\left(\Gamma_{1}\right)^{3}+449571\left(\Gamma_{2}\right)^{4}\left(\Gamma_{1}\right)^{2}+197360\left(\Gamma_{2}\right)^{5}\left(\Gamma_{1}\right)$ $\left.-5600\left(\Gamma_{2}\right)^{6}\right)\left(s^{3}\right)^{8}\left(s^{2}\right)^{6}+2\left(6000\left(\Gamma_{1}\right)^{6}+56155\left(\Gamma_{2}\right)\left(\Gamma_{1}\right)^{5}+220804\left(\Gamma_{2}\right)^{2}\left(\Gamma_{1}\right)^{4}\right.$ $\left.+399698\left(\Gamma_{2}\right)^{3}\left(\Gamma_{1}\right)^{3}+375849\left(\Gamma_{2}\right)^{4}\left(\Gamma_{1}\right)^{2}+211200\left(\Gamma_{2}\right)^{5}\left(\Gamma_{1}\right)+70400\left(\Gamma_{2}\right)^{6}\right)\left(s^{3}\right)^{10}\left(s^{2}\right)^{4}$ $-\left(\Gamma_{1}\right)\left(7200\left(\Gamma_{1}\right)^{5}+76790\left(\Gamma_{2}\right)\left(\Gamma_{1}\right)^{4}+311629\left(\Gamma_{2}\right)^{2}\left(\Gamma_{1}\right)^{3}+575808\left(\Gamma_{2}\right)^{3}\left(\Gamma_{1}\right)^{2}\right.$ $\left.+516909\left(\Gamma_{2}\right)^{4}\left(\Gamma_{1}\right)+189140\left(\Gamma_{2}\right)^{5}\right)\left(s^{3}\right)^{12}\left(s^{2}\right)^{2}+4\left(400\left(\Gamma_{1}\right)^{6}+5175\left(\Gamma_{2}\right)\left(\Gamma_{1}\right)^{5}\right.$ $+21743\left(\Gamma_{2}\right)^{2}\left(\Gamma_{1}\right)^{4}+28301\left(\Gamma_{2}\right)^{3}\left(\Gamma_{1}\right)^{3}-1582\left(\Gamma_{2}\right)^{4}\left(\Gamma_{1}\right)^{2}-23415\left(\Gamma_{2}\right)^{5}\left(\Gamma_{1}\right)$ $\left.\left.-10500\left(\Gamma_{2}\right)^{6}\right)\left(s^{3}\right)^{14}\right)\left(s^{1}\right)^{10}+\left(4\left(300\left(\Gamma_{1}\right)^{6}+6260\left(\Gamma_{2}\right)\left(\Gamma_{1}\right)^{5}-4033\left(\Gamma_{2}\right)^{2}\left(\Gamma_{1}\right)^{4}\right.\right.$ $\left.-19746\left(\Gamma_{2}\right)^{3}\left(\Gamma_{1}\right)^{3}+1767\left(\Gamma_{2}\right)^{4}\left(\Gamma_{1}\right)^{2}-380\left(\Gamma_{2}\right)^{5}\left(\Gamma_{1}\right)+100\left(\Gamma_{2}\right)^{6}\right)\left(s^{2}\right)^{16}$ 
$-6\left(\Gamma_{1}\right)\left(600\left(\Gamma_{1}\right)^{5}+20830\left(\Gamma_{2}\right)\left(\Gamma_{1}\right)^{4}-887\left(\Gamma_{2}\right)^{2}\left(\Gamma_{1}\right)^{3}-19704\left(\Gamma_{2}\right)^{3}\left(\Gamma_{1}\right)^{2}-3867\left(\Gamma_{2}\right)^{4}\left(\Gamma_{1}\right)\right.$ $\left.-5280\left(\Gamma_{2}\right)^{5}\right)\left(s^{3}\right)^{2}\left(s^{2}\right)^{14}+5\left(480\left(\Gamma_{1}\right)^{6}+46976\left(\Gamma_{2}\right)\left(\Gamma_{1}\right)^{5}+18763\left(\Gamma_{2}\right)^{2}\left(\Gamma_{1}\right)^{4}\right.$ $\left.-9994\left(\Gamma_{2}\right)^{3}\left(\Gamma_{1}\right)^{3}-18485\left(\Gamma_{2}\right)^{4}\left(\Gamma_{1}\right)^{2}+2576\left(\Gamma_{2}\right)^{5}\left(\Gamma_{1}\right)-4560\left(\Gamma_{2}\right)^{6}\right)\left(s^{3}\right)^{4}\left(s^{2}\right)^{12}$ $+2\left(1800\left(\Gamma_{1}\right)^{6}-93890\left(\Gamma_{2}\right)\left(\Gamma_{1}\right)^{5}-52081\left(\Gamma_{2}\right)^{2}\left(\Gamma_{1}\right)^{4}+22828\left(\Gamma_{2}\right)^{3}\left(\Gamma_{1}\right)^{3}\right.$ $\left.+36299\left(\Gamma_{2}\right)^{4}\left(\Gamma_{1}\right)^{2}+45080\left(\Gamma_{2}\right)^{5}\left(\Gamma_{1}\right)-6400\left(\Gamma_{2}\right)^{6}\right)\left(s^{3}\right)^{6}\left(s^{2}\right)^{10}-18\left(400\left(\Gamma_{1}\right)^{6}\right.$ $+1200\left(\Gamma_{2}\right)\left(\Gamma_{1}\right)^{5}+15327\left(\Gamma_{2}\right)^{2}\left(\Gamma_{1}\right)^{4}+28654\left(\Gamma_{2}\right)^{3}\left(\Gamma_{1}\right)^{3}+15327\left(\Gamma_{2}\right)^{4}\left(\Gamma_{1}\right)^{2}+1200\left(\Gamma_{2}\right)^{5}\left(\Gamma_{1}\right)$ $\left.+400\left(\Gamma_{2}\right)^{6}\right)\left(s^{3}\right)^{8}\left(s^{2}\right)^{8}+2\left(1800\left(\Gamma_{1}\right)^{6}+104690\left(\Gamma_{2}\right)\left(\Gamma_{1}\right)^{5}+444369\left(\Gamma_{2}\right)^{2}\left(\Gamma_{1}\right)^{4}\right.$ $\left.+743748\left(\Gamma_{2}\right)^{3}\left(\Gamma_{1}\right)^{3}+621229\left(\Gamma_{2}\right)^{4}\left(\Gamma_{1}\right)^{2}+230960\left(\Gamma_{2}\right)^{5}\left(\Gamma_{1}\right)+5600\left(\Gamma_{2}\right)^{6}\right)\left(s^{3}\right)^{10}\left(s^{2}\right)^{6}$ $+5\left(480\left(\Gamma_{1}\right)^{6}-44096\left(\Gamma_{2}\right)\left(\Gamma_{1}\right)^{5}-208917\left(\Gamma_{2}\right)^{2}\left(\Gamma_{1}\right)^{4}-375114\left(\Gamma_{2}\right)^{3}\left(\Gamma_{1}\right)^{3}-338485\left(\Gamma_{2}\right)^{4}\left(\Gamma_{1}\right)^{2}\right.$ $\left.-166512\left(\Gamma_{2}\right)^{5}\left(\Gamma_{1}\right)-43360\left(\Gamma_{2}\right)^{6}\right)\left(s^{3}\right)^{12}\left(s^{2}\right)^{4}+6\left(\Gamma_{1}\right)\left(-600\left(\Gamma_{1}\right)^{5}+17230\left(\Gamma_{2}\right)\left(\Gamma_{1}\right)^{4}\right.$ $\left.+96037\left(\Gamma_{2}\right)^{2}\left(\Gamma_{1}\right)^{3}+180144\left(\Gamma_{2}\right)^{3}\left(\Gamma_{1}\right)^{2}+149377\left(\Gamma_{2}\right)^{4}\left(\Gamma_{1}\right)+47440\left(\Gamma_{2}\right)^{5}\right)\left(s^{3}\right)^{14}\left(s^{2}\right)^{2}$ $+4\left(300\left(\Gamma_{1}\right)^{6}-4460\left(\Gamma_{2}\right)\left(\Gamma_{1}\right)^{5}-30833\left(\Gamma_{2}\right)^{2}\left(\Gamma_{1}\right)^{4}-52986\left(\Gamma_{2}\right)^{3}\left(\Gamma_{1}\right)^{3}-21293\left(\Gamma_{2}\right)^{4}\left(\Gamma_{1}\right)^{2}\right.$ $\left.\left.+17520\left(\Gamma_{2}\right)^{5}\left(\Gamma_{1}\right)+12000\left(\Gamma_{2}\right)^{6}\right)\left(s^{3}\right)^{16}\right)\left(s^{1}\right)^{8}-2\left(2\left(\Gamma_{1}\right)\left(800\left(\Gamma_{1}\right)^{5}+4570\left(\Gamma_{2}\right)\left(\Gamma_{1}\right)^{4}\right.\right.$ $\left.-938\left(\Gamma_{2}\right)^{2}\left(\Gamma_{1}\right)^{3}-6981\left(\Gamma_{2}\right)^{3}\left(\Gamma_{1}\right)^{2}+1737\left(\Gamma_{2}\right)^{4}\left(\Gamma_{1}\right)-490\left(\Gamma_{2}\right)^{5}\right)\left(s^{2}\right)^{18}-5\left(1120\left(\Gamma_{1}\right)^{6}\right.$ $+11100\left(\Gamma_{2}\right)\left(\Gamma_{1}\right)^{5}+5047\left(\Gamma_{2}\right)^{2}\left(\Gamma_{1}\right)^{4}-3550\left(\Gamma_{2}\right)^{3}\left(\Gamma_{1}\right)^{3}+1607\left(\Gamma_{2}\right)^{4}\left(\Gamma_{1}\right)^{2}+64\left(\Gamma_{2}\right)^{5}\left(\Gamma_{1}\right)$ $\left.-160\left(\Gamma_{2}\right)^{6}\right)\left(s^{3}\right)^{2}\left(s^{2}\right)^{16}+\left(\Gamma_{1}\right)\left(4000\left(\Gamma_{1}\right)^{5}+128240\left(\Gamma_{2}\right)\left(\Gamma_{1}\right)^{4}+127693\left(\Gamma_{2}\right)^{2}\left(\Gamma_{1}\right)^{3}\right.$ $\left.+62926\left(\Gamma_{2}\right)^{3}\left(\Gamma_{1}\right)^{2}+37493\left(\Gamma_{2}\right)^{4}\left(\Gamma_{1}\right)+24420\left(\Gamma_{2}\right)^{5}\right)\left(s^{3}\right)^{4}\left(s^{2}\right)^{14}+\left(5600\left(\Gamma_{1}\right)^{6}\right.$ $-171040\left(\Gamma_{2}\right)\left(\Gamma_{1}\right)^{5}-279653\left(\Gamma_{2}\right)^{2}\left(\Gamma_{1}\right)^{4}-230826\left(\Gamma_{2}\right)^{3}\left(\Gamma_{1}\right)^{3}-149413\left(\Gamma_{2}\right)^{4}\left(\Gamma_{1}\right)^{2}$ $\left.-40800\left(\Gamma_{2}\right)^{5}\left(\Gamma_{1}\right)-13600\left(\Gamma_{2}\right)^{6}\right)\left(s^{3}\right)^{6}\left(s^{2}\right)^{12}+\left(-5600\left(\Gamma_{1}\right)^{6}+197360\left(\Gamma_{2}\right)\left(\Gamma_{1}\right)^{5}\right.$ $+449571\left(\Gamma_{2}\right)^{2}\left(\Gamma_{1}\right)^{4}+456432\left(\Gamma_{2}\right)^{3}\left(\Gamma_{1}\right)^{3}+285101\left(\Gamma_{2}\right)^{4}\left(\Gamma_{1}\right)^{2}+83480\left(\Gamma_{2}\right)^{5}\left(\Gamma_{1}\right)$ $\left.+6400\left(\Gamma_{2}\right)^{6}\right)\left(s^{3}\right)^{8}\left(s^{2}\right)^{10}-\left(5600\left(\Gamma_{1}\right)^{6}+230960\left(\Gamma_{2}\right)\left(\Gamma_{1}\right)^{5}+621229\left(\Gamma_{2}\right)^{2}\left(\Gamma_{1}\right)^{4}\right.$ $\left.+743748\left(\Gamma_{2}\right)^{3}\left(\Gamma_{1}\right)^{3}+444369\left(\Gamma_{2}\right)^{4}\left(\Gamma_{1}\right)^{2}+104690\left(\Gamma_{2}\right)^{5}\left(\Gamma_{1}\right)+1800\left(\Gamma_{2}\right)^{6}\right)\left(s^{3}\right)^{10}\left(s^{2}\right)^{8}$ $+\left(5600\left(\Gamma_{1}\right)^{6}+204640\left(\Gamma_{2}\right)\left(\Gamma_{1}\right)^{5}+659547\left(\Gamma_{2}\right)^{2}\left(\Gamma_{1}\right)^{4}+934614\left(\Gamma_{2}\right)^{3}\left(\Gamma_{1}\right)^{3}\right.$ $\left.+659547\left(\Gamma_{2}\right)^{4}\left(\Gamma_{1}\right)^{2}+204640\left(\Gamma_{2}\right)^{5}\left(\Gamma_{1}\right)+5600\left(\Gamma_{2}\right)^{6}\right)\left(s^{3}\right)^{12}\left(s^{2}\right)^{6}+\left(4000\left(\Gamma_{1}\right)^{6}\right.$ $-104240\left(\Gamma_{2}\right)\left(\Gamma_{1}\right)^{5}-453507\left(\Gamma_{2}\right)^{2}\left(\Gamma_{1}\right)^{4}-754554\left(\Gamma_{2}\right)^{3}\left(\Gamma_{1}\right)^{3}-607527\left(\Gamma_{2}\right)^{4}\left(\Gamma_{1}\right)^{2}$ $\left.-244640\left(\Gamma_{2}\right)^{5}\left(\Gamma_{1}\right)-46400\left(\Gamma_{2}\right)^{6}\right)\left(s^{3}\right)^{14}\left(s^{2}\right)^{4}+5\left(\Gamma_{1}\right)\left(-1120\left(\Gamma_{1}\right)^{5}+4380\left(\Gamma_{2}\right)\left(\Gamma_{1}\right)^{4}\right.$ $\left.+33653\left(\Gamma_{2}\right)^{2}\left(\Gamma_{1}\right)^{3}+64862\left(\Gamma_{2}\right)^{3}\left(\Gamma_{1}\right)^{2}+51661\left(\Gamma_{2}\right)^{4}\left(\Gamma_{1}\right)+14792\left(\Gamma_{2}\right)^{5}\right)\left(s^{3}\right)^{16}\left(s^{2}\right)^{2}$ $+2\left(800\left(\Gamma_{1}\right)^{6}+230\left(\Gamma_{2}\right)\left(\Gamma_{1}\right)^{5}-11788\left(\Gamma_{2}\right)^{2}\left(\Gamma_{1}\right)^{4}-26471\left(\Gamma_{2}\right)^{3}\left(\Gamma_{1}\right)^{3}-16648\left(\Gamma_{2}\right)^{4}\left(\Gamma_{1}\right)^{2}\right.$ $\left.\left.+3105\left(\Gamma_{2}\right)^{5}\left(\Gamma_{1}\right)+4500\left(\Gamma_{2}\right)^{6}\right)\left(s^{3}\right)^{18}\right)\left(s^{1}\right)^{6}+\left(\left(\Gamma_{1}\right)^{2}\left(1200\left(\Gamma_{1}\right)^{4}+4640\left(\Gamma_{2}\right)\left(\Gamma_{1}\right)^{3}\right.\right.$ $\left.-3139\left(\Gamma_{2}\right)^{2}\left(\Gamma_{1}\right)^{2}-7078\left(\Gamma_{2}\right)^{3}\left(\Gamma_{1}\right)+3501\left(\Gamma_{2}\right)^{4}\right)\left(s^{2}\right)^{20}-4\left(\Gamma_{1}\right)\left(800\left(\Gamma_{1}\right)^{5}+8960\left(\Gamma_{2}\right)\left(\Gamma_{1}\right)^{4}\right.$ $\left.+2108\left(\Gamma_{2}\right)^{2}\left(\Gamma_{1}\right)^{3}-6809\left(\Gamma_{2}\right)^{3}\left(\Gamma_{1}\right)^{2}+713\left(\Gamma_{2}\right)^{4}\left(\Gamma_{1}\right)+1470\left(\Gamma_{2}\right)^{5}\right)\left(s^{3}\right)^{2}\left(s^{2}\right)^{18}-4\left(3700\left(\Gamma_{1}\right)^{6}\right.$ $-17180\left(\Gamma_{2}\right)\left(\Gamma_{1}\right)^{5}-7211\left(\Gamma_{2}\right)^{2}\left(\Gamma_{1}\right)^{4}+19338\left(\Gamma_{2}\right)^{3}\left(\Gamma_{1}\right)^{3}+8169\left(\Gamma_{2}\right)^{4}\left(\Gamma_{1}\right)^{2}-1800\left(\Gamma_{2}\right)^{5}\left(\Gamma_{1}\right)$ $\left.-600\left(\Gamma_{2}\right)^{6}\right)\left(s^{3}\right)^{4}\left(s^{2}\right)^{16}+2\left(\Gamma_{1}\right)\left(46400\left(\Gamma_{1}\right)^{5}+33760\left(\Gamma_{2}\right)\left(\Gamma_{1}\right)^{4}+80327\left(\Gamma_{2}\right)^{2}\left(\Gamma_{1}\right)^{3}\right.$ $\left.+157154\left(\Gamma_{2}\right)^{3}\left(\Gamma_{1}\right)^{2}+84607\left(\Gamma_{2}\right)^{4}\left(\Gamma_{1}\right)+24420\left(\Gamma_{2}\right)^{5}\right)\left(s^{3}\right)^{6}\left(s^{2}\right)^{14}-5\left(43360\left(\Gamma_{1}\right)^{6}\right.$ $+93648\left(\Gamma_{2}\right)\left(\Gamma_{1}\right)^{5}+156325\left(\Gamma_{2}\right)^{2}\left(\Gamma_{1}\right)^{4}+180906\left(\Gamma_{2}\right)^{3}\left(\Gamma_{1}\right)^{3}+99765\left(\Gamma_{2}\right)^{4}\left(\Gamma_{1}\right)^{2}$ $\left.+29936\left(\Gamma_{2}\right)^{5}\left(\Gamma_{1}\right)+4560\left(\Gamma_{2}\right)^{6}\right)\left(s^{3}\right)^{8}\left(s^{2}\right)^{12}+4\left(70400\left(\Gamma_{1}\right)^{6}+211200\left(\Gamma_{2}\right)\left(\Gamma_{1}\right)^{5}\right.$ $+375849\left(\Gamma_{2}\right)^{2}\left(\Gamma_{1}\right)^{4}+399698\left(\Gamma_{2}\right)^{3}\left(\Gamma_{1}\right)^{3}+220804\left(\Gamma_{2}\right)^{4}\left(\Gamma_{1}\right)^{2}+56155\left(\Gamma_{2}\right)^{5}\left(\Gamma_{1}\right)$ $\left.+6000\left(\Gamma_{2}\right)^{6}\right)\left(s^{3}\right)^{10}\left(s^{2}\right)^{10}-5\left(43360\left(\Gamma_{1}\right)^{6}+166512\left(\Gamma_{2}\right)\left(\Gamma_{1}\right)^{5}+338485\left(\Gamma_{2}\right)^{2}\left(\Gamma_{1}\right)^{4}\right.$ $\left.+375114\left(\Gamma_{2}\right)^{3}\left(\Gamma_{1}\right)^{3}+208917\left(\Gamma_{2}\right)^{4}\left(\Gamma_{1}\right)^{2}+44096\left(\Gamma_{2}\right)^{5}\left(\Gamma_{1}\right)-480\left(\Gamma_{2}\right)^{6}\right)\left(s^{3}\right)^{12}\left(s^{2}\right)^{8}$ $+2\left(46400\left(\Gamma_{1}\right)^{6}+244640\left(\Gamma_{2}\right)\left(\Gamma_{1}\right)^{5}+607527\left(\Gamma_{2}\right)^{2}\left(\Gamma_{1}\right)^{4}+754554\left(\Gamma_{2}\right)^{3}\left(\Gamma_{1}\right)^{3}\right.$ $\left.+453507\left(\Gamma_{2}\right)^{4}\left(\Gamma_{1}\right)^{2}+104240\left(\Gamma_{2}\right)^{5}\left(\Gamma_{1}\right)-4000\left(\Gamma_{2}\right)^{6}\right)\left(s^{3}\right)^{14}\left(s^{2}\right)^{6}-4\left(3700\left(\Gamma_{1}\right)^{6}\right.$

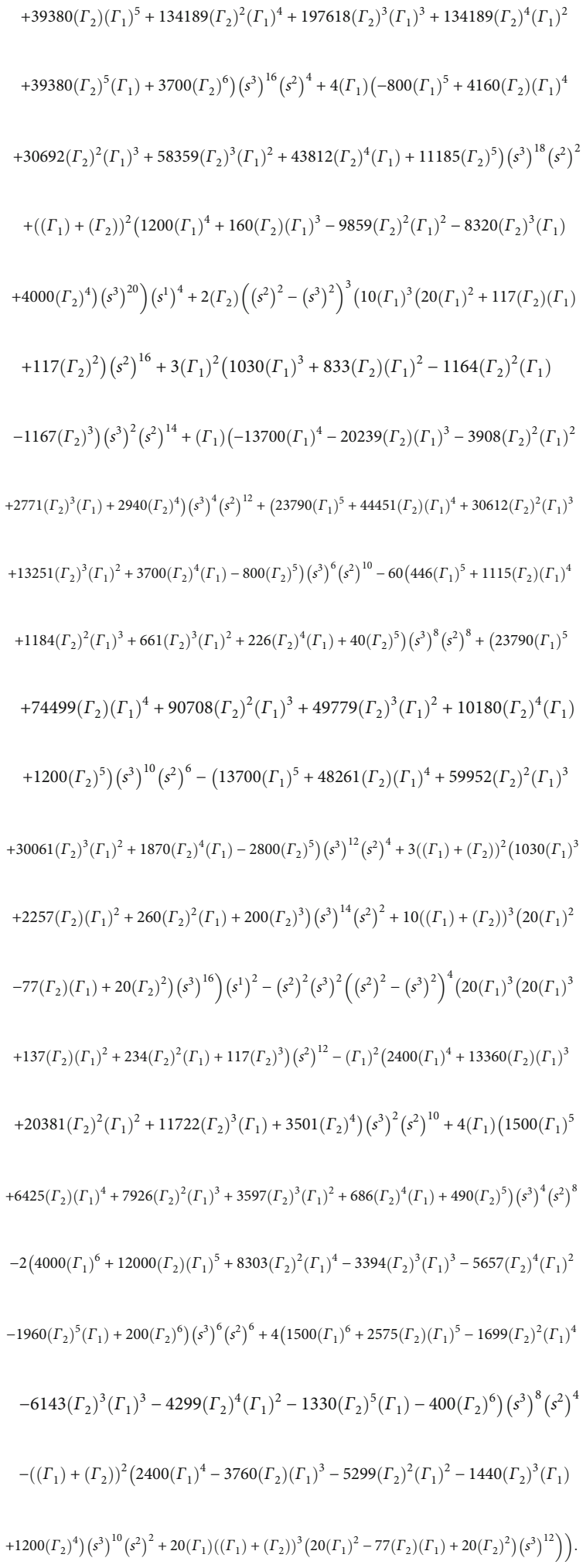

\section{Details of Other KCC invariants}

In this section, we describe in detail the first, third, fourth, and fifth KCC invariants on the assumptions of self-similarity, and $\left(\Gamma_{1}, \Gamma_{2}, \Gamma_{3}\right)=(2 \Gamma, 2 \Gamma,-\Gamma)$. The components of the 
first invariant $E^{i}$ are given by

$$
\begin{gathered}
E^{1}=\frac{3 \Gamma^{2}}{4 \pi^{2}\left(s^{1}\right)^{3}\left(s^{2}\right)^{4}\left(s^{3}\right)^{4}}\left[-\left(s^{1}\right)^{4}\left(s^{2}\right)^{2}\left(s^{3}\right)^{2}+\left(s^{1}\right)^{2}\left(s^{2}\right)^{6}+\left(s^{1}\right)^{6}\left(s^{2}\right)^{2}\right. \\
-5\left(s^{1}\right)^{2}\left(s^{3}\right)^{6}+6\left(s^{1}\right)^{4}\left(s^{3}\right)^{4}-2\left(s^{1}\right)^{6}\left(s^{3}\right)^{2}+7\left(s^{2}\right)^{6}\left(s^{3}\right)^{2} \\
\left.-7\left(s^{2}\right)^{4}\left(s^{3}\right)^{4}+\left(s^{2}\right)^{2}\left(s^{3}\right)^{6}-2\left(s^{2}\right)^{8}+\left(s^{3}\right)^{8}\right] \\
E^{2}=-\frac{3 \Gamma^{2}}{4 \pi^{2}\left(s^{1}\right)^{4}\left(s^{2}\right)^{3}\left(s^{3}\right)^{4}}\left[\left(s^{1}\right)^{2}\left(s^{2}\right)^{4}\left(s^{3}\right)^{2}-\left(s^{1}\right)^{6}\left(s^{2}\right)^{2}\right. \\
-\left(s^{1}\right)^{2}\left(s^{2}\right)^{6}-7\left(s^{1}\right)^{6}\left(s^{3}\right)^{2}+7\left(s^{1}\right)^{4}\left(s^{3}\right)^{4}-\left(s^{1}\right)^{2}\left(s^{3}\right)^{6}+2\left(s^{1}\right)^{8} \\
\left.+5\left(s^{2}\right)^{2}\left(s^{3}\right)^{6}-6\left(s^{2}\right)^{4}\left(s^{3}\right)^{4}+2\left(s^{2}\right)^{6}\left(s^{3}\right)^{2}-\left(s^{3}\right)^{8}\right] \\
E^{3}=\frac{3 \Gamma^{2}}{16 \pi^{2}\left(s^{1}\right)^{4}\left(s^{2}\right)^{4}\left(s^{3}\right)^{3}}\left[-10\left(s^{1}\right)^{2}\left(s^{2}\right)^{2}\left(s^{3}\right)^{4}+4\left(s^{1}\right)^{6}\left(s^{2}\right)^{2}\right. \\
+2\left(s^{1}\right)^{4}\left(s^{2}\right)^{4}+4\left(s^{1}\right)^{2}\left(s^{2}\right)^{6}+16\left(s^{1}\right)^{6}\left(s^{3}\right)^{2} \\
-15\left(s^{1}\right)^{4}\left(s^{3}\right)^{4}+4\left(s^{1}\right)^{2}\left(s^{3}\right)^{6}-5\left(s^{1}\right)^{8}+4\left(s^{2}\right)^{2}\left(s^{3}\right)^{6} \\
\left.-15\left(s^{2}\right)^{4}\left(s^{3}\right)^{4}+16\left(s^{2}\right)^{6}\left(s^{3}\right)^{2}-5\left(s^{2}\right)^{8}\right] .
\end{gathered}
$$

Moreover, all components of the third, fourth, and fifth invariants are zero:

$$
B_{j k}^{i}=0, \quad B_{l j k}^{i}=0, \quad D_{j k l}^{i}=0 .
$$

\section{Data Availability}

No data were used to support this study.

\section{Conflicts of Interest}

The authors declare that there is no conflict of interest regarding the publication of this paper.

\section{References}

[1] D. D. Kosambi, "Parallelism and path-spaces," Mathematische Zeitschrift, vol. 37, no. 1, pp. 608-618, 1933.

[2] E. Cartan and D. D. Kosambi, "Observations sur le mémoire précédent," Mathematische Zeitschrift, vol. 37, no. 1, pp. 619-622, 1933.

[3] S. S. Chern, "Sur la géométrie d'un système d'équations différentielles du second ordre," Bulletin des Sciences Mathematiques, vol. 63, pp. 206-212, 1939.

[4] P. L. Antonelli, R. S. Ingarden, and M. Matsumoto, The Theory of Sprays and Finsler Spaces with Applications in Physics and Biology, Kluwer, Dordrecht, 1993.

[5] P. L. Antonelli and I. Bucataru, "Volterra-Hamilton production models with discounting: general theory and worked examples," Nonlinear Analysis: Real World Applications, vol. 2, no. 3, pp. 337-356, 2001.

[6] P. L. Antonelli, L. Bevilacqua, and S. F. Rutz, "Theories and models in symbiogenesis," Nonlinear Analysis: Real World Applications, vol. 4, no. 5, pp. 743-753, 2003.
[7] P. L. Antonelli and S. F. Rutz, "Finslerian Volterra-Hamilton systems in Clementsian forest succession," Nonlinear Analysis: Real World Applications, vol. 6, no. 5, pp. 899-913, 2005.

[8] P. L. Antonelli, S. F. Rutz, and K. T. Fonseca, "Carbon flux models in the Mata-Atlântica rain forest of Brazil," Nonlinear Analysis: Real World Applications, vol. 11, no. 5, pp. 35243531, 2010.

[9] P. L. Antonelli, S. F. Rutz, and C. E. Hirakawa, "The mathematical theory of endosymbiosis I," Nonlinear Analysis: Real World Applications, vol. 12, no. 6, pp. 3238-3251, 2011.

[10] K. Yamasaki and T. Yajima, "Lotka-Volterra system and KCC theory: Differential geometric structure of competitions and predations," Nonlinear Analysis: Real World Applications, vol. 14, no. 4, pp. 1845-1853, 2013.

[11] K. Yamasaki and T. Yajima, "Differential geometric structure of non-equilibrium dynamics in competition and predation: Finsler geometry and KCC theory," Journal of Dynamical Systems and Geometric Theories, vol. 14, no. 2, pp. 137-153, 2016.

[12] V. S. Sabău, "Systems biology and deviation curvature tensor," Nonlinear Analysis: Real World Applications, vol. 6, no. 3, pp. 563-587, 2005.

[13] M. K. Gupta and C. K. Yadav, "KCC theory and its application in a tumor growth model," Mathematical Methods in the Applied Sciences, vol. 40, no. 18, pp. 7470-7487, 2017.

[14] S. V. Sabau, "Some remarks on Jacobi stability," Nonlinear Analysis: Theory, Methods \& Applications, vol. 63, no. 5-7, pp. e143-e153, 2005.

[15] T. Yajima and K. Yamasaki, "Jacobi stability for dynamical systems of two-dimensional second-order differential equations and application to overhead crane system," International Journal of Geometric Methods in Modern Physics, vol. 13, no. 4, article 1650045, 2016.

[16] S. Oiwa and T. Yajima, "Jacobi stability analysis and chaotic behavior of nonlinear double pendulum," International Journal of Geometric Methods in Modern Physics, vol. 14, no. 12, article 1750176, 2017.

[17] T. Harko, C. Y. Ho, C. S. Leung, and S. Yip, "Jacobi stability analysis of the Lorenz system," International Journal of Geometric Methods in Modern Physics, vol. 12, no. 7, article 1550081, 2015.

[18] T. Yajima and H. Nagahama, "KCC-theory and geometry of the Rikitake system," Journal of Physics A: Mathematical and Theoretical, vol. 40, no. 11, pp. 2755-2772, 2007.

[19] T. Yajima and H. Nagahama, "Nonlinear dynamical systems and KCC-theory," Acta Mathematica Academiae Paedagogicae Nyí Regyháziensis. New Series, vol. 24, no. 1, pp. 179-189, 2008.

[20] T. Yajima and H. Nagahama, "Geometrical invariants of Rikitake system in KCC-theory," Tensor. New Series, vol. 69, pp. 106-113, 2008.

[21] T. Yajima and H. Nagahama, "Geometrical unified theory of Rikitake system and KCC-theory," Nonlinear Analysis: Theory, Methods \& Applications, vol. 71, no. 12, pp. e203-e210, 2009.

[22] T. Yajima and H. Nagahama, "Tangent bundle viewpoint of the Lorenz system and its chaotic behavior," Physics Letters A, vol. 374, no. 11-12, pp. 1315-1319, 2010.

[23] T. Harko and V.S. Sabau, "Jacobi stability of the vacuum in the static spherically symmetric brane world models," Physical Review D, vol. 77, no. 10, article 104009, 2008. 
[24] C. G. Böhmer and T. Harko, "Nonlinear stability analysis of the Emden-Fowler equation," Journal of Nonlinear Mathematical Physics, vol. 17, no. 4, pp. 503-516, 2010.

[25] C. G. Böhmer, T. Harko, and S. V. Sabau, "Jacobi stability analysis of dynamical systems-applications in gravitation and cosmology," Advances in Theoretical and Mathematical Physics, vol. 16, no. 4, pp. 1145-1196, 2012.

[26] M. J. Lake and T. Harko, "Dynamical behavior and Jacobi stability analysis of wound strings," The European Physical Journal C, vol. 76, no. 6, 2016.

[27] M. K. Gupta and C. K. Yadav, "Rabinovich-Fabrikant system in view point of KCC theory in Finsler geometry," Journal of Interdisciplinary Mathematics, vol. 22, no. 3, pp. 219-241, 2019.

[28] M. K. Gupta, C. K. Yadav, and A. K. Gupta, “A geometrical study of Wang-Chen system in view of KCC theory," TWMS Journal of Applied and Engineering Mathematics, vol. 10, no. 4, pp. 1064-1073, 2020.

[29] H. Abolghasem, "Jacobi stability of Hamiltonian systems," International Journal of Pure and Apllied Mathematics, vol. 87, no. 1, pp. 181-194, 2013.

[30] H. Aref, "Motion of three vortices," Physics of Fluids, vol. 22, no. 3, pp. 393-400, 1979.

[31] H. Aref, N. Rott, and H. Thomann, "Gröbli's solution of the three-vortex problem," Annual Review of Fluid Mechanics, vol. 24, no. 1, pp. 1-21, 1992.

[32] H. Aref, "Point vortex dynamics: a classical mathematics playground," Journal of Mathematical Physics, vol. 48, no. 6, article 065401, 2007.

[33] H. Aref, "Self-similar motion of three point vortices," Physics of Fluids, vol. 22, no. 5, article 057104, 2010.

[34] G. Badin and A. M. Barry, "Collapse of generalized Euler and surface quasigeostrophic point vortices," Physical Review E, vol. 98, no. 2, article 023110, 2018.

[35] Y. Kimura, "Similarity solution of two-dimensional point vortices," Journal of the Physical Society of Japan, vol. 56, no. 6, pp. 2024-2030, 1987.

[36] P. K. Newton, The N-Vortex Problem: Analytical Techniques, Springer, New York, 2010.

[37] P. L. Antonelli and I. Bucataru, "KCC theory of a system of second order differential equations," in Handbook of Finsler Geometry, vol. 1, P. L. Antonelli, Ed., pp. 83-174, Kluwer, Dordrecht, 2003.

[38] P. L. Antonelli and I. Bucataru, "New results about the geometric invariants in KCC-theory," Analele Stiintifice ale Universitatii Al I Cuza din Iasi - Matematica, vol. 47, no. 2, pp. 405420, 2001.

[39] A. G. Kurosh, Higher algebra, MIR Publishers, Moscow, 1972. 